
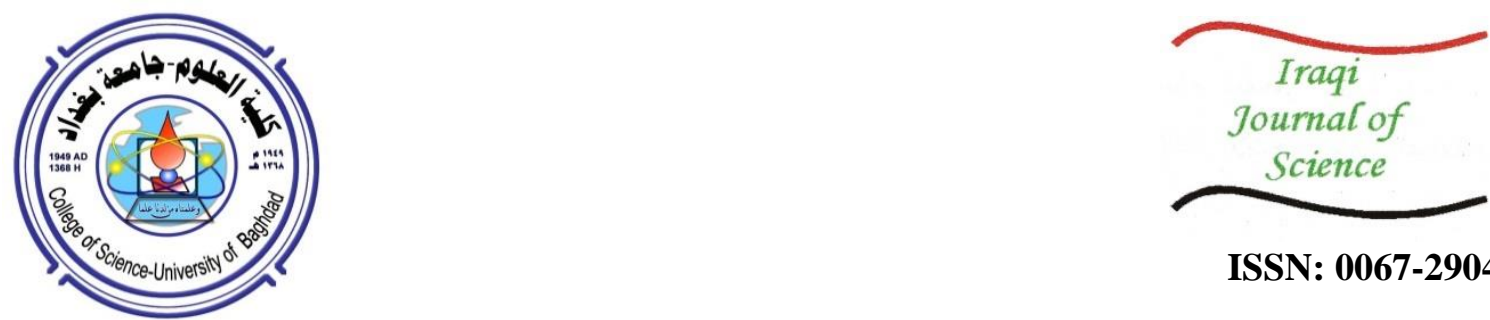

ISSN: 0067-2904

\title{
The Dynamics of a Prey-Predator Model with Infectious Disease in Prey: Role of Media Coverage
}

\author{
Wassan Hussein*, Huda Abdul Satar \\ Department of Mathematics, College of Science, University of Baghdad, Baghdad, IRAQ
}

Received: $21 / 4 / 2021$

Accepted: 9/6/2021

\begin{abstract}
:
In this paper, an eco-epidemiological model with media coverage effect is proposed and studied. A prey-predator model with modified Leslie-Gower and functional response is studied. An SIS-type of disease in prey is considered. The existence, uniqueness and boundedness of the solution of the model are discussed. The local and global stability of this system are carried out. The conditions for the persistence of all species are established. The local bifurcation in the model is studied. Finally, numerical simulations are conducted to illustrate the analytical results.
\end{abstract}

Keywords: Media coverage, Leslie-Gower, disease, stability, persistence, bifurcation.

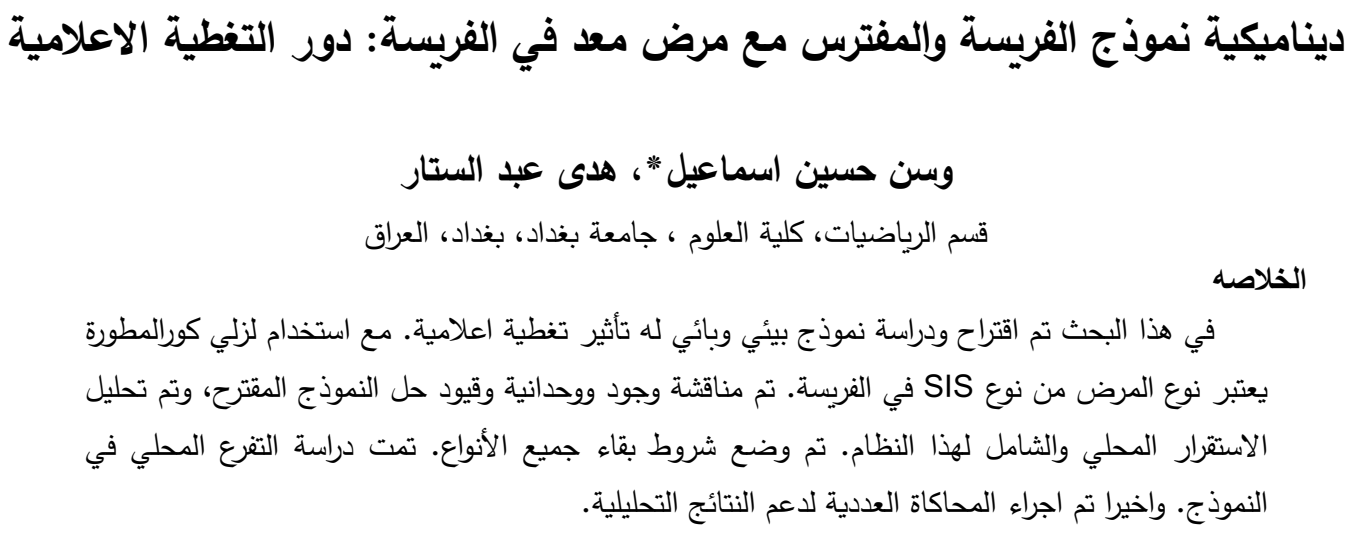

\section{Introduction}

The utilization of mathematical models for studying and understanding the spread and controlling infectious diseases has become a highly important tool. The scientists extensively studied the dynamics of ecological models in the existence of infectious diseases and provided important insights into complex biological processes. The study of the spread of infectious diseases within populations of ecological systems is resulting in a branch called eco-epidemiology.

This subject is rapidly growing as a branch of theoretical ecology ([1]-[3]), Later on, several researchers were proposed and studied eco-epidemiological models involving many biological factors, see ([4-13])

The modified Leslie-Gower prey-predator model, which is proposed by Leslie and Gower [14] and modified by May [15], is considered by many scientists [16-19]). In the modified

*Email: wasanhussain95@gmail.com 
Leslie-Gower model, the predator acts as a generalist predator because it avoids extinction by using an alternative source of food. Although in case of a severe scarcity of prey the predator population growth may still be limited by the fact that their favorite food is not available in abundance, some predator species can switch to another available food in the environment. The impact of media coverage is one of the most key factors to establish the prevention and control measure that affect the spread of infectious disease. The role of media coverage of disease outbreaks is therefore crucial and should be given prominence in the study of disease dynamics [20].

Liu and Cui [21] studied a container model that characterized the spread and control of infectious disease under the influence of media coverage. Tchuenche and Bauch [22] proposed and studied a susceptible-infected-hospitalized-recovered model with vital dynamics, where media coverage of disease incidence and prevalence can influence people to reduce their contact rates. $\mathrm{Li}$ and $\mathrm{Cui}[23]$ introduced constant and pulse vaccines in media coverage for SIS disease models. In recent years, attempts have been made to develop mathematical models for the transmission dynamics of infectious diseases within the ecoepidemiological model. Alwan and Abdul Satar [24] proposed and studied a prey-predator model having a disease in predator species and involving media coverage. They used it for describing the predation process as a Lotka-Volterra type of functional response.

In this paper, an SIS-type of disease in prey is considered, so that a modified Leslie-Gower prey-predator model is proposed and the effect of media coverage on the dynamics of a proposed eco-epidemiological model is studied. Moreover, Lotka-Volterra type of functional response is used to describe the predation process. The organization of this paper is given as follows. Section (2) deals with the model formulation. Section (3) determines the equilibrium points (EPs) and analyzes their local stability. The global stability for the EPs is studied with the help of the Lyapunov method (LM) in section (4). While, the bifurcation analysis of the system is investigated in section (5). Section (6) deals with the numerical simulation of the system. Finally, the discussion and conclusions are addressed in section (7).

\section{The mathematical model}

In this section, the effect of media coverage on a modified Leslie-Gower prey-predator model is formulated mathematically. An infectious disease of SIS type in prey species is included in the model. It is assumed that the prey is consumed by the predator according to Lotka-Volterra type of functional responses. Now, in order to represent the dynamics of such a real-world system, the following hypotheses are adopted.

Let the variables $S(T), I(T)$, and $Y(T)$ represent the densities at time $T$ for the susceptible prey, infected prey, and predator, respectively. It is assumed that $S(T)$ grows logistically with $r_{1}>0$ as an intrinsic growth rate, while $I(T)$ cannot reproduce due to the disease, instead of that, it competes with the susceptible prey for environment carrying capacity $K_{1}>0$. However, the predator $Y(T)$ grows logistically with $r_{2}>0$ as a growth rate by sexual reproduction and carrying capacity, depending on the prey and given by $K_{2}+S+I$, where $K_{2}>0$ represents a residual loss in predator population. The predator species $Y(T)$ consumes both the prey species $S(T)$ and $I(T)$ using Lotka-Volterra type of functional responses with maximum attack rates of $a_{1}>0$ and $a_{2}>0$, respectively. The term $\left(b_{1}-\frac{n I}{m+I}\right)$ represents the infection rate due to the direct contact between $S(T)$ and $I(T)$, where $b_{1}>0$ is the contact rate before media coverage alert, while $\frac{n I}{m+I}$ represents the reduced value in the contact due to media coverage alert, so that $n>0$ is the maximum transmission rate under the media coverage and $m>0$ is the non-response rate of individual to the media coverage. Furthermore, since it is well known that the media coverage cannot prevent the spreading of the disease completely, then from now onward we take $b_{1} \geq n$. Also, the infected individuals may recover with a rate of $\alpha>0$. The disease-caused death rate of infected individuals is 
given by $d_{1}>0$, while the parameter $p>0$ is the maximum value in which per capita reduction rate of predator species can be attained due to intra-specific. Finally, the infected prey causes predator death due to disease when feeding on it with a probability $\varepsilon \in(0,1)$.

According to the above hypotheses, the dynamics of the above-described system, that is consisting of a diseased prey-predator system incorporating the media coverage, can be represented by the following set of differential equations:

$$
\begin{aligned}
& \frac{d S}{d T}=r_{1}\left(1-\frac{S+I}{K_{1}}\right) S-\left(b_{1}-\frac{n I}{m+I}\right) S I+\alpha I-a_{1} S Y, \\
& \frac{d I}{d T}=\left(b_{1}-\frac{n I}{m+I}\right) S I-\left(\alpha+d_{1}\right) I-a_{2} I Y, \\
& \frac{d Y}{d T}=r_{2} Y\left(1-\frac{p Y}{K_{2}+S+I}\right)-\varepsilon a_{2} I Y,
\end{aligned}
$$

with $S(0) \geq 0, I(0) \geq 0$ and $Y(0) \geq 0$ as an initial condition. Therefore, the system (1) has the domain $\mathbb{R}_{+}^{3}=\left\{(S, I, Y) \in \mathbb{R}^{3} \mid X \geq 0, S \geq 0, I \geq 0\right\}$.

Clearly, the system (1) contains a $C^{1}$ functions; therefore, these functions are Lipschitzain. Hence, the solution of the system (1) exists and is unique. Further, the uniformly bounded of the solutions of the system (1) is proved in the following theorem.

Theorem (1): The system (1) has uniformly bounded solutions.

Proof. Define $W_{1}=S+I$, then $\frac{d W_{1}}{d T}$ can be written as

$\frac{d W_{1}}{d T} \leq r_{1}\left(1-\frac{S+I}{K_{1}}\right) S-d_{1} I \leq 2 r_{1} S-\frac{r_{1} S^{2}}{K_{1}}-\mu W_{1}$,

where $\mu=\min \left\{r_{1}, d_{1}\right\}$. Then, direct computation shows that for $T$ goes to $\infty$, we have $W_{1} \leq \ell_{1}$, where $\ell_{1}=\frac{r_{1} K_{1}}{\mu}$.

Since the third equation of predator is a logistic growth equation, then it is easy to verify that $Y \leq \frac{r_{2}\left(K_{2}+\ell_{1}\right)}{4 p}=\ell_{2}$. Therefore, all the variables are bounded.

\section{Existence of EPs and Their Local Stability Analysis}

The existence of EPs of the system (1) and their local stability analysis are discussed. The existence conditions for each of these EPs are established.

The trivial EP, represented by $P_{0}=(0,0,0)$, always exists.

The first axial EP, represented by $P_{1}=\left(K_{1}, 0,0\right)$, always exists as the susceptible prey population grows to carrying capacity in the absence of predation.

The second axial EP, represented by $P_{2}=\left(0,0, \frac{K_{2}}{p}\right)$, always exists as the predator population that growth logistically grows to carrying capacity supplied by the environment in the absence of preferred prey.

The predator-free EP is denoted by $P_{3}=(\bar{S}, \bar{I}, 0)$, where

$$
\bar{S}=\frac{(m+\bar{I})\left(\alpha+d_{1}\right)}{\left[b_{1} m+\left(b_{1}-n\right) \bar{I}\right]}
$$

where $\bar{I}$ represents a positive root for the third order polynomial equation:

where

$$
B_{1} I^{3}+B_{2} I^{2}+B_{3} I+B_{4}=0,
$$

$$
\begin{aligned}
B_{1}= & -\left(b_{1}-n\right)\left[r_{1}\left(\alpha+d_{1}\right)+K_{1} d_{1}\left(b_{1}-n\right)\right]<0, \\
B_{2}= & {\left[r_{1} K_{1}\left(\alpha+d_{1}\right)\left(b_{1}-n\right)-r_{1}\left(\alpha+d_{1}\right)^{2}-r_{1} m\left(\alpha+d_{1}\right)\left(b_{1}-n\right)-\right.} \\
& \left.r_{1}\left(\alpha+d_{1}\right) b_{1}-2 K_{1} d_{1} b_{1} m\left(b_{1}-n\right)\right], \\
B_{3}= & {\left[r_{1} K_{1} m\left(b_{1}-n\right)+r_{1} K_{1}\left(\alpha+d_{1}\right) b_{1} m-2 r_{1} m\left(\alpha+d_{1}\right)^{2}-\right.} \\
\left.r_{1} m^{2} b_{1}\left(\alpha+d_{1}\right)-K_{1} d_{1} m^{2} b_{1}^{2}\right], & \\
B_{4}= & {\left[r_{1} m\left(\alpha+d_{1}\right)\left(K_{1} b_{1}-m\left(\alpha+d_{1}\right)\right] .\right.}
\end{aligned}
$$

Therefore, $P_{3}=(\bar{S}, \bar{I}, 0)$ exists if 


$$
\begin{gathered}
B_{4}>0 \text { and } B_{3}>0 . \\
\quad O R \\
B_{4}>0 \text { and } B_{2}<0 .
\end{gathered}
$$

(2c) The infected prey-free EP is represented by $P_{4}=(\widetilde{S}, 0, \tilde{Y})$, where

$$
\tilde{S}=\frac{K_{1}\left(r_{1} p-a_{1} K_{2}\right)}{\left(r_{1} p+a_{1} K_{2}\right)}, \quad \text { and } \quad \tilde{Y}=\frac{K_{2}+\tilde{S}}{p},
$$

(3a) which exists provided that

$$
r_{1} p>a_{1} K_{2}
$$

The coexistence or positive EP is denoted by $P_{5}=\left(S^{*}, I^{*}, Y^{*}\right)$, where

$$
Y^{*}=\frac{\left(r_{2}-\varepsilon a_{2} I^{*}\right)\left(K_{2}+S^{*}+I^{*}\right)}{p}
$$

(4) where the point $\left(S^{*}, I^{*}\right)$ represents the positive intersection point of the following two isoclines:

$$
\begin{aligned}
& g_{1}(S, I)=r_{1}\left(1-\frac{S+I}{K_{1}}\right) S-\left(b_{1}-\frac{n I}{m+I}\right) S I+\alpha I-a_{1} S\left(\frac{\left(r_{2}-\varepsilon a_{2} I\right)\left(K_{2}+S+I\right)}{p}\right)=0 \\
& g_{2}(S, I)=\left(b_{1}-n \frac{I}{m+I}\right) S-\left(\alpha+d_{1}\right)-a_{2}\left(\frac{\left(r_{2}-\varepsilon a_{2} I\right)\left(K_{2}+S+I\right)}{p}\right)=0
\end{aligned}
$$

Obviously, as $I \rightarrow 0$, then the isoclines become

$$
\begin{aligned}
& g_{1}(S, I)=r_{1}\left(1-\frac{S}{K_{1}}\right) S-a_{1} S \frac{r_{2}\left(K_{2}+S\right)}{p}=0 . \\
& g_{2}(S, I)=b_{1} S-\left(\alpha+d_{1}\right)-a_{2} \frac{r_{2}\left(K_{2}+S\right)}{p}=0 .
\end{aligned}
$$

(5b) Therefore, $g_{1}(S)$ intersects the $S$-axis at the positive point $s_{1}=\frac{K_{1}\left(p r_{1}-a_{1} r_{2} K_{2}\right)}{p r_{1}+a_{1} r_{2} K_{1}}$; however, $g_{2}(S)$ intersects the $S$-axis at the positive point $s_{2}=\frac{p\left(\alpha+d_{1}\right)+a_{2} r_{2} K_{2}}{b_{1} p-a_{2} r_{2}}$. Hence, the two isoclines (5a) have a unique positive intersection point and then $P_{5}$ exists uniquely in the interior of $\mathbb{R}_{+}^{3}$ if

$$
\begin{gathered}
\frac{p}{r_{2}}>\max \left\{\frac{a_{1} K_{2}}{r_{1}}, \frac{a_{2}}{b_{1}}\right\} . \\
S_{2}>S_{1} . \\
\frac{d I}{d S}=-\frac{\partial g_{1} / \partial S}{\partial g_{1} / \partial I}>0 . \\
\frac{d I}{d S}=-\frac{\partial g_{2} / \partial S}{\partial g_{2} / \partial I}<0 .
\end{gathered}
$$

Now, to establish the local stability, the Jacobian matrix (JM) of system (1) about arbitrary point $(S, I, Y)$ is

$$
J(X, S, I)=\left[\rho_{i j}\right]_{3 \times 3}
$$

where $\quad \rho_{11}=r_{1}-\frac{2 r_{1} S+r_{1} I}{K_{1}}-\left(b_{1}-\frac{n I}{m+I}\right) I-a_{1} Y, \quad \rho_{12}=-\frac{r_{1} S}{K_{1}}-b_{1} S+\frac{2 n S I}{m+I}-\frac{n s I^{2}}{(m+I)^{2}}+\alpha$, $\rho_{13}=-a_{1} S, \quad \rho_{21}=\left(b_{1}-\frac{n I}{m+I}\right) I, \quad \rho_{22}=b_{1} S-\frac{2 n S I}{m+I}+\frac{n s I^{2}}{(m+I)^{2}}-\left(\alpha+d_{1}\right)-a_{2} Y, \quad \rho_{23}=$ $-a_{2} I, \rho_{31}=\frac{r_{2} p Y^{2}}{\left(K_{2}+S+I\right)^{2}}, \rho_{32}=\frac{r_{2} p Y^{2}}{\left(K_{2}+S+I\right)^{2}}-\varepsilon a_{2} Y, \rho_{33}=r_{2}-\frac{2 r_{2} p Y}{\left(K_{2}+S+I\right)}-\varepsilon a_{2} I$.

It is clear that the system (1) has JM at trivial EP, $P_{0}=(0,0,0)$ specified by

$$
J\left(P_{0}\right)=\left[\begin{array}{ccc}
r_{1} & \alpha & 0 \\
0 & -\left(\alpha+d_{1}\right) & 0 \\
0 & 0 & r_{2}
\end{array}\right],
$$

(7a) Therefore, the eigenvalues of $J\left(P_{0}\right)$ are:

$$
\lambda_{01}=r_{1}, \lambda_{02}=-\left(\alpha+d_{1}\right), \lambda_{03}=r_{2}
$$

Hence, the trivial EP is unstable (saddle point).

The JM of the system (1) at the first axial EP, $P_{1}=\left(K_{1}, 0,0\right)$ is 


$$
J\left(P_{1}\right)=\left[\begin{array}{ccc}
-r_{1} & -r_{1}-b_{1} K_{1}+\alpha & -a_{1} K_{1} \\
0 & b_{1} K_{1}-\left(\alpha+d_{1}\right) & 0 \\
0 & 0 & r_{2}
\end{array}\right],
$$

(8a) Therefore, the eigenvalues of $J\left(P_{1}\right)$ are given by

$$
\lambda_{11}=-r_{1}<0, \lambda_{12}=b_{1} K_{1}-\left(\alpha+d_{1}\right), \lambda_{13}=r_{2}>0 \text {. }
$$

Hence, the first axial EP, $P_{1}=\left(K_{1}, 0,0\right)$ is unstable (saddle point).

The JM of the system (1) at the second axial EP, $P_{2}=\left(0,0, \frac{K_{2}}{p}\right)$ is

$$
J\left(P_{2}\right)=\left[\begin{array}{ccc}
r_{1}-\frac{a_{1} K_{2}}{p} & \alpha & 0 \\
0 & -\left(\alpha+d_{1}\right)-\frac{a_{2} K_{2}}{p} & 0 \\
\frac{r_{2}}{p} & \frac{r_{2}-\varepsilon a_{2} K_{2}}{p} & -r_{2}
\end{array}\right] .
$$

Clearly, the eigenvalues of $J\left(P_{2}\right)$ are given by

$$
\lambda_{21}=r_{1}-\frac{a_{1} K_{2}}{p}, \lambda_{22}=-\left(\alpha+d_{1}\right)-\frac{a_{2} K_{2}}{p} \text {, and } \lambda_{23}=-r_{2} \text {. }
$$

Hence, all the eigenvalues are negative, and the second axial EP is LAS provided that

$$
r_{1}<\frac{a_{1} K_{2}}{p}
$$

Now the JM of the system (1) at the predator-free EP, $P_{3}=(\bar{S}, \bar{I}, 0)$, can be written as:

$$
J\left(P_{3}\right)=\left[a_{i j}\right]_{3 \times 3},
$$

where $\quad a_{11}=r_{1}-\frac{2 r_{1} \bar{S}+r_{1} \bar{I}}{K_{1}}-\left(b_{1}-\frac{n \bar{I}}{m+\bar{I}}\right) \bar{I}, \quad a_{12}=-\frac{r_{1} \bar{S}}{K_{1}}-b_{1} \bar{S}+\frac{2 n \bar{S} \bar{I}}{m+\bar{I}}-\frac{n s \bar{I}^{2}}{(m+\bar{I})^{2}}+\alpha, \quad a_{13}=$ $-a_{1} \bar{S}, a_{21}=\left(b_{1}-\frac{n \bar{I}}{m+\bar{I}}\right) \bar{I}, a_{22}=b_{1} \bar{S}-\frac{2 n \bar{S} \bar{I}}{m+\bar{I}}+\frac{n s \bar{I}^{2}}{(m+\bar{I})^{2}}-\left(\alpha+d_{1}\right), a_{23}=-a_{2} \bar{I}, a_{31}=a_{32}=$ 0 , and $a_{33}=r_{2}-\varepsilon a_{2} \bar{I}$.

Clearly, one of the eigenvalues is $\lambda_{33}=r_{2}-\varepsilon a_{2} \bar{I}$ and the other two eigenvalues are the roots of the equation:

$$
\lambda_{3}^{2}-T_{2} \lambda_{3}+D_{2}=0
$$

(10b) where $T_{2}=a_{11}+a_{22}$ and $D_{2}=\left(a_{11} a_{22}-a_{12} a_{21}\right)$. Note that the direct computation gives that the roots (eigenvalues) of the equation (10b) can be written as

$$
\lambda_{31}=\frac{T_{2}}{2}+\frac{1}{2} \sqrt{T_{2}{ }^{2}-4 D_{2}} ; \lambda_{32}=\frac{T_{2}}{2}-\frac{1}{2} \sqrt{T_{2}{ }^{2}-4 D_{2}}
$$

Hence, all the eigenvalues of $J\left(P_{3}\right)$ have negative real parts and hence $P_{3}$ is LAS if and only if

$$
\begin{aligned}
r_{2} & <\varepsilon a_{2} \bar{I}, \\
\frac{2 n \bar{S} \bar{I}}{m+\bar{I}}+\alpha & <\frac{r_{1} \bar{S}}{K_{1}}+b_{1} \bar{S}+\frac{n s \bar{I}^{2}}{\left(m+\bar{I}^{2}\right.}, \\
r_{1} & <\frac{2 r_{1} \bar{S}+r_{1} \bar{I}}{K_{1}}+\left(b_{1}-\frac{n \bar{I}}{m+\bar{I}}\right) \bar{I}, \\
b_{1} \bar{S} & +\frac{n s \bar{I}^{2}}{(m+\bar{I})^{2}}<\frac{2 n \bar{S} \bar{I}}{m+\bar{I}}+\left(\alpha+d_{1}\right) .
\end{aligned}
$$

Now the JM of the system (1) at the infected prey-free EP, $P_{4}=(\widetilde{S}, 0, \tilde{Y})$, can be written as:

$$
J\left(P_{4}\right)=\left[\begin{array}{ccc}
r_{1}-\frac{2 r_{1} \tilde{S}}{K_{1}}-a_{1} \tilde{Y} & -\frac{r_{1} \tilde{S}}{K_{1}}-b_{1} \tilde{S}+\alpha & -a_{1} \tilde{S} \\
0 & b_{1} \tilde{S}-\left(\alpha+d_{1}\right)-a_{2} \tilde{Y} & 0 \\
\frac{r_{2}}{p} & \frac{r_{2}}{p}-\varepsilon a_{2} \tilde{Y}^{2} & -r_{2}
\end{array}\right] .
$$

Clearly, one of the eigenvalues is $\lambda_{42}=b_{1} \tilde{S}-\left(\alpha+d_{1}\right)-a_{2} \tilde{Y}$ and the other two eigenvalues are given by:

$$
\lambda_{41}=\frac{T_{3}}{2}+\frac{1}{2} \sqrt{T_{3}{ }^{2}-4 D_{3}} ; \lambda_{43}=\frac{T_{3}}{2}-\frac{1}{2} \sqrt{T_{3}{ }^{2}-4 D_{3}},
$$

where $T_{3}=r_{1}-\frac{2 r_{1} \tilde{S}}{K_{1}}-a_{1} \tilde{Y}-r_{2}$, and $D_{3}=-\left(r_{1}-\frac{2 r_{1} \tilde{S}}{K_{1}}-a_{1} \tilde{Y}\right) r_{2}+a_{1} \tilde{S} \frac{r_{2}}{p}$. 
Therefore, all the eigenvalues have negative real parts, and then $P_{4}$ is LAS if and only if

$$
\begin{aligned}
b_{1} \tilde{S} & <\left(\alpha+d_{1}\right)+a_{2} \tilde{Y}, \\
r_{1} & <\frac{2 r_{1} \tilde{S}}{K_{1}}+a_{1} \tilde{Y} .
\end{aligned}
$$

Finally the JM evaluated at the positive EP, $P_{5}$, is given by:

$$
J\left(P_{5}\right)=\left[e_{i j}\right]_{3 \times 3}
$$

where $e_{11}=r_{1}-\frac{2 r_{1} S^{*}+r_{1} I^{*}}{K_{1}}-\left(b_{1}-\frac{n I^{*}}{m+I^{*}}\right) I^{*}-a_{1} Y^{*}$,

$e_{12}=-\frac{r_{1} S^{*}}{K_{1}}-b_{1} S^{*}+\frac{2 n S^{*} I^{*}}{m+I^{*}}-\frac{n s I^{* 2}}{\left(m+I^{*}\right)^{2}}+\alpha, e_{13}=-a_{1} S^{*}$,

$e_{21}=\left(b_{1}-\frac{n I^{*}}{m+I^{*}}\right) I^{*}, e_{22}=b_{1} S^{*}-\frac{2 n S^{*} I^{*}}{m+I^{*}}+\frac{n I^{* 2}}{\left(m+I^{*}\right)^{2}}-\left(\alpha+d_{1}\right)-a_{2} Y^{*}$,

$e_{23}=-a_{2} I^{*}, e_{31}=\frac{r_{2} p Y^{* 2}}{\left(K_{2}+S^{*}+I^{*}\right)^{2}}, e_{32}=\frac{r_{2} p Y^{* 2}}{\left(K_{2}+S^{*}+I^{*}\right)^{2}}-\varepsilon a_{2} Y^{*}$.

$e_{33}=r_{2}-\frac{2 r_{2} p Y^{*}}{\left(K_{2}+S^{*}+I^{*}\right)}-\varepsilon a_{2} I^{*}$.

Then the characteristic equation of $J\left(P_{5}\right)$ can be written as:

$\lambda_{5}^{3}+M_{1} \lambda_{5}^{2}+M_{2} \lambda_{5}+M_{3}=0$

with

$$
\begin{aligned}
M_{1} & =-\left(e_{11}+e_{22}+e_{33}\right)=-\Gamma_{1}, \\
M_{2} & =\left(e_{11} e_{22}-e_{12} e_{21}\right)+\left(e_{11} e_{33}-e_{13} e_{31}\right)+\left(e_{22} e_{33}-e_{23} e_{32}\right) \\
& =\Gamma_{2}+\Gamma_{3}+\Gamma_{4} \\
M_{3} & =-\left[e_{33}\left(e_{11} e_{22}-e_{12} e_{21}\right)+e_{23}\left(e_{12} e_{31}-e_{11} e_{32}\right)+e_{13}\left(e_{21} e_{32}-e_{22} e_{31}\right)\right] \\
& =-\left[e_{33} \Gamma_{2}+e_{23} \Gamma_{5}+e_{13} \Gamma_{6}\right],
\end{aligned}
$$

$$
\begin{aligned}
\Delta & =M_{1} M_{2}-M_{3}=-\Gamma_{7} \Gamma_{2}-\Gamma_{8} \Gamma_{3}-\Gamma_{9} \Gamma_{4}-2 \Gamma_{11}+\Gamma_{10}, \\
\text { where } \Gamma_{1} & =\left(e_{11}+e_{22}+e_{33}\right) ; \Gamma_{2}=\left(e_{11} e_{22}+e_{12} e_{21}\right) ; \Gamma_{3}=\left(e_{11} e_{33}+e_{13} e_{31}\right) \\
\Gamma_{4} & =\left(e_{22} e_{33}-e_{23} e_{32}\right) ; \Gamma_{5}=\left(e_{12} e_{31}-e_{11} e_{32}\right) ; \Gamma_{6}=\left(e_{21} e_{32}-e_{22} e_{31}\right) \\
\Gamma_{7} & =\left(e_{11}+e_{22}\right) ; \Gamma_{8}=\left(e_{11}+e_{33}\right) ; \Gamma_{9}=\left(e_{22}+e_{33}\right) \\
\Gamma_{10} & =e_{12} e_{23} e_{31}+e_{13} e_{21} e_{32} ; \Gamma_{11}=e_{11} e_{22} e_{33}
\end{aligned}
$$

Accordingly, the local stability of the positive EP can be given in the following theorem.

Theorem (2). The positive EP of the system (1) is locally LAS provided that the following conditions hold:

$$
\begin{aligned}
& r_{1}<\frac{2 r_{1} S^{*}+r_{1} I^{*}}{K_{1}}+\left(b_{1}-\frac{n I^{*}}{m+I^{*}}\right) I^{*}+a_{1} Y^{*}, \\
& b_{1} S^{*}+\frac{n S I^{* 2}}{\left(m+I^{*}\right)^{2}}<\frac{2 n S^{*} I^{*}}{m+I^{*}}+\left(\alpha+d_{1}\right)+a_{2} Y^{*}, \\
& r_{2}<\frac{2 r_{2} p Y^{*}}{\left(K_{2}+S^{*}+I^{*}\right)}+\varepsilon a_{2} I^{*} \\
& \quad \Gamma_{10}<2 \Gamma_{11} .
\end{aligned}
$$

Proof. According to the Routh- Hurwitz criterion, all roots of the characteristic equation given by Eq. (14b) have negative real parts roots, if and only if $M_{1}>0, \quad M_{3}>0$, and $\Delta=M_{1} M_{2}-M_{3}>0$.

Straightforward computation shows that the conditions (15a)-(15d) satisfy the Routh-Hurwitz criterion conditions, and hence all the eigenvalues of the Eq. (14b) have negative real parts. Then, the positive EP is LAS.

The persistence of the system (1) is studied. It is well known that the biological system is persistent if and only if all its species are persistent all the time. Now, according to the system (1), if the predator individuals disappear, then 


$$
\begin{aligned}
& \frac{d S}{d T}=r_{1}\left(1-\frac{S+I}{K_{1}}\right) S-\left(b_{1}-\frac{n I}{m+I}\right) S I+\alpha I=g_{1}(S, I) \\
& \frac{d I}{d T}=\left(b_{1}-n \frac{I}{m+I}\right) S I-\left(\alpha+d_{1}\right) I=g_{2}(S, I)
\end{aligned}
$$

Clearly, subsystem (16) is a 2D space that has a unique positive point given by $(\bar{S}, \bar{I})$, which are given by Eq. (2a), and exists uniquely in the $S I$-plane under the condition (2c). By using Poincare Bendixon theorem, the solution of system (16) approaches either to EP $(\bar{S}, \bar{I})$ or else to the periodic dynamics. Now, by using the continuous function $u(S, I)=\frac{1}{S I}$, we obtain

$$
\nabla=\frac{\partial\left(u g_{1}\right)}{\partial S}+\frac{\partial\left(u g_{2}\right)}{\partial I}=-\frac{r_{1}}{K_{1} I}-\frac{\alpha}{S^{2}}-\frac{n m}{(m+I)^{2}}<0
$$

Therefore, according to the Dulac criterion, there is no periodic dynamics in the interior of the positive quadrant of $S I$-plane. Hence, using Poincare Bendixon theorem, the positive EP of the subsystem (16) is globally asymptotically stable (GAS) whenever it exists. Then the system (1) has no periodic dynamics in the boundary $S I-$ plane.

The following theorem explains the conditions that guarantee the persistence of the system.

Theorem 3. The system (1) is uniformly persistent if

$$
\left.\begin{array}{c}
\frac{a_{1} K_{2}}{p}<r_{1}, \\
\varepsilon a_{2} \bar{I}<r_{2}, \\
\left(\alpha+d_{1}\right)+a_{2} \tilde{Y}<b_{1} \tilde{S} \\
o r \\
\frac{2 r_{1} \tilde{S}}{K_{1}}+a_{1} \tilde{Y}<r_{1}
\end{array}\right\} .
$$

(17c) Proof: Suppose that $\sigma$ is a point in the interior of $\mathbb{R}_{+}^{3}$ and $\varphi(\sigma)$ is the orbit through $\sigma$, and let $\Upsilon(\sigma)$ is the omega limit set of $\varphi(\sigma)$. Further, since $\Upsilon(\sigma)$ is bounded, due to the boundedness of the system (1), then we first show that $P_{0} \notin \Upsilon(\sigma)$.

Assume the contrary, since $P_{0}$ is a saddle point, then by Butler-McGhee lemma [25], there is at least one other point $\sigma_{1}$ such that $\sigma_{1} \in \omega^{S}\left(P_{0}\right) \cap \Upsilon(\sigma)$, where $\omega^{s}\left(P_{0}\right)$ is the stable manifold of $P_{0}$.

Now, since the stable manifold of $P_{0}$ is given by $Y$-direction and the entire orbit through $\sigma_{1}$, say $\varphi\left(\sigma_{1}\right)$, is contained in $\Upsilon(\sigma)$, then we obtain a contradiction to the boundedness of $\Upsilon(\sigma)$, due to the containment of an unbounded positive axis in it. This shows that $P_{0} \notin \Upsilon(\sigma)$.

Now, to proof that $P_{1} \notin \Upsilon(\sigma)$, we assume the converse. Since $P_{1}$ is a saddle point, then by Butler-McGhee lemma, there is another point, say $\sigma_{2}$, so that $\sigma_{2} \in \omega^{S}\left(P_{1}\right) \cap \Upsilon(\sigma)$. Now, since the stable manifold of $P_{1}$ is given by $S$-direction and the entire orbit through $\sigma_{2}$, say $\gamma\left(\sigma_{2}\right)$, is contained in $\Upsilon(\sigma)$, hence we obtain a contradiction to the boundedness of $\Upsilon(\sigma)$, due to the containment of an unbounded positive axis in it. This shows that $P_{1} \notin \Upsilon(\sigma)$.

Now, since the points $P_{2}, P_{3}$ and $P_{4}$ are saddle points under the conditions (17a), (17b), and $(17 \mathrm{c})$, respectively. Then by using similar argument as that given in the first part of the proof, we obtain that $P_{2}, P_{3}$ and $P_{4} \notin \Upsilon(\sigma)$.

\section{Global Stability Analysis}

In this section, the global stability (GS) is studied for all LS Eps. Lyapunov method is used to investigate the GS or specify the basin of attraction of each EP.

Theorem 4. Assume that the second axial EP, $P_{2}=(0,0, \overline{\bar{Y}})$, of the system (1) is LAS in $\mathbb{R}_{+}^{3}$, and:

$$
\begin{aligned}
r_{1}< & \frac{r_{2} p \overline{\bar{Y}}^{2}}{K_{2}\left(K_{2}+\ell_{1}\right)}, \\
& \frac{r_{2} p \overline{\bar{Y}}}{K_{2}{ }^{2}}<\min \left\{a_{1}, a_{2}(1+\varepsilon)\right\}, \\
\varepsilon a_{2} \overline{\bar{Y}}< & d_{1}+\frac{r_{2} p \bar{Y}^{2}}{K_{2}\left(K_{2}+\ell_{1}\right)},
\end{aligned}
$$


where $\ell_{1}$ is the upper bound of $S+I$. Then, it is GAS in $\mathbb{R}_{+}^{3}$.

Proof: Consider the following function:

$L_{1}=S+I+\left(Y-\overline{\bar{Y}}-\ln \frac{Y}{\overline{\bar{Y}}}\right)$.

Then, $L_{1}$ is a $C^{1}$ real valued function, which is a positive definite. Now, the derivative $\frac{d L_{1}}{d T}$ can be calculated as:

$$
\begin{aligned}
\frac{d L_{1}}{d T} \leq & r_{1} S-a_{1} S Y-a_{2} I Y-d_{1} I-\frac{r_{2} p}{\left(K_{2}+S+I\right)}(Y-\overline{\bar{Y}})^{2} \\
& +\frac{r_{2} p \overline{\bar{Y}} S(Y-\bar{Y})}{K_{2}\left(K_{2}+S+I\right)}+\frac{r_{2} p \overline{\bar{Y}} I(Y-\bar{Y})}{K_{2}\left(K_{2}+S+I\right)}-\varepsilon a_{2} I Y+\varepsilon a_{2} \overline{\bar{Y}} I .
\end{aligned}
$$

Hence,

$$
\begin{gathered}
\frac{d L_{1}}{d T} \leq\left[r_{1}-\frac{r_{2} p \overline{\bar{Y}}^{2}}{K_{2}\left(K_{2}+S+I\right)}\right] S-\frac{r_{2} p}{\left(K_{2}+S+I\right)}(Y-\overline{\bar{Y}})^{2} \\
-\left[a_{1}-\frac{r_{2} p \overline{\bar{Y}}}{K_{2}\left(K_{2}+S+I\right)}\right] S Y-\left[a_{2}(1+\varepsilon)-\frac{r_{2} p \overline{\bar{Y}}}{K_{2}\left(K_{2}+S+I\right)}\right] I Y \\
-\left[d_{1}+\frac{r_{2} p \overline{\bar{Y}}^{2}}{K_{2}\left(K_{2}+S+I\right)}-\varepsilon a_{2} \overline{\bar{Y}}\right] I .
\end{gathered}
$$

Therefore, by using the above set of conditions, it is obtained that:

$$
\begin{gathered}
\frac{d L_{1}}{d T}<\left[r_{1}-\frac{r_{2} p \bar{Y}^{2}}{K_{2}\left(K_{2}+S+I\right)}\right] S-\frac{r_{2} p}{\left(K_{2}+S+I\right)}(Y-\overline{\bar{Y}})^{2} \\
-\left[d_{1}+\frac{r_{2} p \overline{\bar{Y}}^{2}}{K_{2}\left(K_{2}+S+I\right)}-\varepsilon a_{2} \overline{\bar{Y}}\right] I .
\end{gathered}
$$

Obviously, $\frac{d L_{1}}{d T}$ is a negative definite, and since $L_{1}$ is radially unbounded function, then $P_{2}$ is GAS.

Theorem 5. Assume that the predator-free EP, $P_{3}$, of the system (1) is LAS in $\mathbb{R}_{+}^{3}$, and:

$$
\begin{aligned}
& {\left[\frac{r_{1}}{K_{1}}-\frac{n m \bar{I}}{A \bar{A}}-\frac{\alpha}{S}\right]^{2}<4\left[\frac{r_{1}}{K_{1}}+\frac{\alpha \bar{I}}{S \bar{S}}\right] \frac{n m \bar{S}}{A \bar{A}}} \\
& {\left[a_{1} \bar{S}+a_{2} \bar{I}+r_{2}\right] \ell_{2}<\left[\sqrt{\frac{r_{1}}{K_{1}}+\frac{\alpha \bar{I}}{S \bar{S}}}(S-\bar{S})+\sqrt{\frac{n m \bar{S}}{A \bar{A}}}(I-\bar{I})\right]^{2} .}
\end{aligned}
$$

where all the symbols are defined in the proof. Then it is GAS in $\mathbb{R}_{+}^{3}$.

Proof: Consider the following function:

$L_{2}=\left(S-\bar{S}-\bar{S} \ln \frac{S}{\bar{S}}\right)+\left(I-\bar{I}-\bar{I} \ln \frac{I}{\bar{I}}\right)+Y$

Clearly, $L_{2}: \mathbb{R}_{+}^{3} \rightarrow \mathbb{R}$ is $C^{1}$ function that is positive definite. Then we have

$$
\begin{gathered}
\frac{d L_{2}}{d T} \leq-\left[\frac{r_{1}}{K_{1}}+\frac{\alpha \bar{I}}{S \bar{S}}\right](S-\bar{S})^{2}-\left[\frac{r_{1}}{K_{1}}-\frac{n m \bar{I}}{A \bar{A}}-\frac{\alpha}{S}\right](S-\bar{S})(I-\bar{I}) \\
-\frac{n m \bar{S}}{A \bar{A}}(I-\bar{I})^{2}+\left[a_{1} \bar{S}+a_{2} \bar{I}+r_{2}\right] \ell_{2},
\end{gathered}
$$

where $A=m+I, \bar{A}=m+\bar{I}$, and $\ell_{2}$ is the upper bound of $Y$. Therefore, by using the condition (19a), it is obtained that

$$
\frac{d L_{2}}{d T} \leq-\left[\sqrt{\frac{r_{1}}{K_{1}}+\frac{\alpha \bar{I}}{S \bar{S}}}(S-\bar{S})+\sqrt{\frac{n m \bar{S}}{A \bar{A}}}(I-\bar{I})\right]^{2}+\left[a_{1} \bar{S}+a_{2} \bar{I}+r_{2}\right] \ell_{2} .
$$

Obviously, under the condition (19b), we have $\frac{d L_{2}}{d T}$ is negative definite, and since $L_{2}$ is radially unbounded function, then $P_{3}$ is GAS.

Theorem 6. Assume that the infected prey-free EP, $P_{4}$, of the system (1) is LAS in $\mathbb{R}_{+}^{3}$, and:

$$
\begin{aligned}
& {\left[a_{1}-\frac{r_{2}}{K_{2}+S+I}\right]^{2}<4\left(\frac{r_{1}}{K_{1}}\right)\left(\frac{r_{2} p}{K_{2}+\ell_{1}}\right),} \\
& \frac{r_{2}}{K_{2}}<a_{2}(1+\varepsilon),
\end{aligned}
$$




$$
\left(b_{1}+n\right) \tilde{S}+\frac{r_{1} \tilde{S}}{K_{1}}+\varepsilon a_{2} \tilde{Y}<\frac{r_{2}\left(K_{2}+\tilde{S}\right)}{p\left(K_{2}+\ell_{1}\right)}+d_{1} .
$$

Then, it is GAS in $\mathbb{R}_{+}^{3}$.

Proof: Consider the following function:

$$
L_{3}=\left(S-\tilde{S}-\tilde{S} \ln \frac{S}{\tilde{S}}\right)+I+\left(Y-\tilde{Y}-\tilde{Y} \ln \frac{Y}{\tilde{Y}}\right)
$$

Clearly, $L_{3}: \mathbb{R}_{+}^{3} \rightarrow \mathbb{R}$ is $C^{1}$ that is a positive definite real valued function.

$$
\begin{aligned}
\frac{d L_{3}}{d T} \leq & -\frac{r_{1}}{K_{1}}(S-\tilde{S})^{2}-\left[a_{1}-\frac{r_{2}}{K_{2}+S+I}\right](S-\tilde{S})(Y-\tilde{Y}) \\
& -\frac{r_{2} p}{K_{2}+\ell_{1}}(Y-\tilde{Y})^{2}-\left[a_{2}(\varepsilon+1)-\frac{r_{2}}{K_{2}}\right] I Y \\
- & {\left[\frac{r_{2}\left(K_{2}+\tilde{S}\right)}{p\left(K_{2}+\ell_{1}\right)}+d_{1}-\left(b_{1}+n\right) \tilde{S}-\frac{r_{1} \tilde{S}}{K_{1}}-\varepsilon a_{2} \tilde{Y}\right] I, }
\end{aligned}
$$

where $\ell_{1}$ is the upper bound for $S+I$. Using conditions (20a)-(20b), its obtained that:

$$
\begin{aligned}
\frac{d L_{3}}{d T}< & -\left[\sqrt{\frac{r_{1}}{K_{1}}}(S-\tilde{S})+\sqrt{\frac{r_{2} p}{K_{2}+\ell_{1}}}(Y-\tilde{Y})\right]^{2} \\
& -\left[\frac{r_{2}\left(K_{2}+\tilde{S}\right)}{p\left(K_{2}+\ell_{1}\right)}+d_{1}-\left(b_{1}+n\right) \tilde{S}-\frac{r_{1} \tilde{S}}{K_{1}}-\varepsilon a_{2} \tilde{Y}\right] I .
\end{aligned}
$$

Obviously, under the condition (20c), it is obtained that $\frac{d L_{3}}{d T}$ is negative definite. Further, since $L_{3}$ is radially unbounded function, then $P_{4}$ is GAS.

Theorem 7. Assume that the positive EP, $P_{5}=\left(S^{*}, I^{*}, Y^{*}\right)$, of the system (1) is LAS in $\mathbb{R}_{+}^{3}$, and:

$$
\left.\begin{array}{l}
\delta_{12}^{2}<\delta_{11} \delta_{22} \\
\delta_{13}^{2}<\delta_{11} \delta_{33} \\
\delta_{23}^{2}<\delta_{22} \delta_{33}
\end{array}\right\}
$$

where all the symbols are defined in the proof. Then, it is GAS in $\mathbb{R}_{+}^{3}$.

Proof: Consider the following function:

$$
L_{4}=\left(S-S^{*}-S^{*} \ln \frac{S}{S^{*}}\right)+\left(I-I^{*}-I^{*} \ln \frac{I}{I^{*}}\right)+\left(Y-Y^{*}-Y \ln \frac{Y}{Y^{*}}\right) .
$$

Clearly, $L_{4}: \mathbb{R}_{+}^{3} \rightarrow \mathbb{R}$ is a $C^{1}$ function that is a positive definite. Then, we have

$$
\begin{gathered}
\frac{d L_{4}}{d T}=-\left[\frac{r_{1}}{K_{1}}+\frac{\alpha I^{*}}{S S^{*}}\right]\left(S-S^{*}\right)^{2}-\left[\frac{r_{1}}{K_{1}}-\frac{n I I^{*}}{A A^{*}}-\frac{\alpha}{S}\right]\left(S-S^{*}\right)\left(I-I^{*}\right) \\
-\frac{n m S^{*}}{A A^{*}}\left(I-I^{*}\right)^{2}-\left[a_{2}(\varepsilon+1)-\frac{r_{2} p Y^{*}}{B B^{*}}\right]\left(I-I^{*}\right)\left(Y-Y^{*}\right) \\
-\frac{r_{2} p}{B}\left(Y-Y^{*}\right)^{2}-\left[a_{1}-\frac{r_{2} p Y^{*}}{B B^{*}}\right]\left(S-S^{*}\right)\left(Y-Y^{*}\right)
\end{gathered}
$$

where $A=m+I, A^{*}=m+I^{*}, B=K_{2}+S+I$, and $B^{*}=K_{2}+S^{*}+I^{*}$. So, after using the given conditions (21), it is obtained that:

$$
\begin{aligned}
\frac{d L_{4}}{d T} \leq-\frac{1}{2} & {\left[\sqrt{\delta_{11}}\left(S-S^{*}\right)+\sqrt{\delta_{22}}\left(I-I^{*}\right)\right]^{2} } \\
-\frac{1}{2} & {\left[\sqrt{\delta_{11}}\left(S-S^{*}\right)+\sqrt{\delta_{33}}\left(Y-Y^{*}\right)\right]^{2} } \\
& \quad-\frac{1}{2}\left[\sqrt{\delta_{22}}\left(I-I^{*}\right)-\sqrt{\delta_{33}}\left(Y-Y^{*}\right)\right]^{2},
\end{aligned}
$$

here $\delta_{11}=\left[\frac{r_{1}}{k_{1}}+\frac{\alpha I^{*}}{S S^{*}}\right], \delta_{22}=\frac{n m S^{*}}{A A^{*}}, \delta_{33}=\frac{r_{2} p}{B}, \delta_{12}=\left[\frac{r_{1}}{K_{1}}-\frac{n I I^{*}}{A A^{*}}-\frac{\alpha}{S}\right], \delta_{13}=\left[a_{1}-\frac{r_{2} p Y^{*}}{B B^{*}}\right]$ and $\delta_{23}=\left[a_{2}(\varepsilon+1)-\frac{r_{2} p Y^{*}}{B B^{*}}\right]$.

Obviously, we have $\frac{d L_{4}}{d T}$ is negative definite. Also, since $L_{4}$ is radially unbounded function, then $P_{5}$ is GAS. 


\section{Local bifurcation}

The effect of varying the parameters values on the dynamics of the system (1) is studied in this section using the local bifurcation analysis with the help of the Sotomayor's theorem.

Now, for simplifying the notations, rewrite the system (1) in the vector form as follows $\frac{d X}{d T}=F(X)$, with $\boldsymbol{X}=(S, I, Y)^{t}$ and $F=\left(G_{1}, G_{2}, G_{3}\right)^{t}$

So, according to the JM of the system (1) at the point $(S, I, Y)$, it is easy to verify that for any vector $U=\left(u_{1}, u_{2}, u_{3}\right)^{t}$, we have that

$$
D^{2} \boldsymbol{F}(\boldsymbol{X})(U, U)=\left[\begin{array}{l}
\sum_{i, j=1}^{3} \frac{\partial G_{1}}{\partial x_{i} \partial x_{j}}\left(u_{i}\right)\left(u_{j}\right) \\
\sum_{i, j=1}^{3} \frac{\partial G_{2}}{\partial x_{i} \partial x_{j}}\left(u_{i}\right)\left(u_{j}\right) \\
\sum_{i, j=1}^{3} \frac{\partial G_{3}}{\partial x_{i} \partial x_{j}}\left(u_{i}\right)\left(u_{j}\right)
\end{array}\right]=\left[b_{i j}\right]_{3 \times 1},
$$

$2 a_{1} u_{1} u_{3}$

where $\quad b_{11}=-\frac{2 r_{1}}{K_{1}} u_{1}^{2}-\left(\frac{2 r_{1}}{K_{1}}+2 b_{1}-\frac{4 n I}{m+I}+\frac{2 n I^{2}}{(m+I)^{2}}\right) u_{1} u_{2}+\left(\frac{2 m n S}{(m+I)^{2}}-\frac{2 m n S I}{(m+I)^{3}}\right) u_{2}^{2}-$

$b_{21}=\left(2 b_{1}-\frac{4 n I}{m+I}+\frac{2 n I^{2}}{(m+I)^{2}}\right) u_{1} u_{2}-\left(\frac{2 m n S}{(m+I)^{2}}-\frac{2 m n S I}{(m+I)^{3}}\right) u_{2}^{2}-2 a_{2} u_{2} u_{3}$

$b_{31}=-\frac{2 r_{2} p Y^{2}}{\left(K_{2}+S+I\right)^{3}} u_{1}^{2}-\frac{4 r_{2} p Y^{2}}{\left(K_{2}+S+I\right)^{3}} u_{1} u_{2}+\frac{4 r_{2} p Y}{\left(K_{2}+S+I\right)^{2}} u_{1} u_{3}-\frac{2 r_{2} p Y^{2}}{\left(K_{2}+S+I\right)^{3}} u_{2}^{2}-\frac{2 r_{2} p}{K_{2}+S+I} u_{3}^{2}+$ $\left(\frac{4 r_{2} p Y}{\left(K_{2}+S+I\right)^{2}}-\quad 2 \varepsilon a_{2}\right) u_{2} u_{3}$.

We also have

where

$$
D^{3} \boldsymbol{F}(\boldsymbol{X})(U, U, U)=\left[c_{i j}\right]_{3 \times 1}
$$

$$
\begin{aligned}
& c_{11}=\left[\frac{2 m n}{(m+I)^{2}}\left(3-\frac{I}{(m+I)}\right)-\frac{2 n I}{(m+I)^{2}}\left(1-\frac{2 I}{(m+I)}\right)\right] u_{1} u_{2}^{2}-\frac{6 m n S}{(m+I)^{2}}\left(1-\frac{2 I}{(m+I)^{2}}\right) u_{2}^{3} \\
& c_{21}=\left[\frac{-2 m n}{(m+I)^{2}}\left(3-\frac{I}{(m+I)}\right)+\frac{2 n I}{(m+I)^{2}}\left(1-\frac{2 I}{(m+I)}\right)\right] u_{1} u_{2}^{2}+\frac{6 m n S}{(m+I)^{2}}\left(1-\frac{2 I}{(m+I)^{2}}\right) u_{2}^{3} \\
& c_{31}=\frac{6 r_{2} p Y^{2}}{\left(K_{2}+S+I\right)^{4}}\left(u_{1}+u_{2}\right)^{3}-\frac{12 r_{2} p Y u_{3}}{\left(K_{2}+S+I\right)^{3}}\left(u_{1}+u_{2}\right)^{2}+\frac{6 r_{2} p}{\left(K_{2}+S+I\right)^{2}}\left(u_{1}+u_{2}\right)
\end{aligned}
$$

The occurrence of LB around the EPs, $P_{2}, P_{3}, P_{4}$ and $P_{5}$, is investigated respectively.

Theorem 8. Assume that the parameter $r_{1}$ satisfies that

$$
r_{1} \equiv r_{1}^{*}=\frac{a_{1} K_{2}}{p}
$$

(24) Then, the system (1) near the second axial EP, $P_{2}$, has a transcritical bifurcation (TB) but saddle - node bifurcation (SNB) and a pitchfork bifurcation (PB) cannot occur.

Proof: Note that, when $r_{1}=r_{1}{ }^{*}$, then the JM of the system (1) at $P_{2}$ can be written as

$J_{1}=J\left(P_{2}, r_{1}{ }^{*}\right)=\left[\begin{array}{ccc}0 & \alpha & 0 \\ 0 & -\left(\alpha+d_{1}\right)-\frac{a_{2} K_{2}}{p} & 0 \\ \frac{r_{2}}{p} & \frac{r_{2}-\varepsilon a_{2} K_{2}}{p} & -r_{2}\end{array}\right]$.

So, $J_{1}$ has the following eigenvalues: $\lambda_{11}^{*}=0, \lambda_{12}^{*}=-\left(\alpha+d_{1}\right)-\frac{a_{2} K_{2}}{p}<0$ and $\lambda_{12}^{*}=-r_{2}$. Hence, the second axial EP is a non-hyperbolic point, and then the necessary but not sufficient condition for bifurcation is satisfied.

Let $\boldsymbol{U}_{1}=\left(u_{11}, u_{12}, u_{13}\right)^{t}$ be the eigenvectors of $J_{1}$ of $\lambda_{11}^{*}=0$. Then, simple computation gives that $\boldsymbol{U}_{1}=\left(u_{11}, 0, \gamma_{1} u_{11}\right)^{t}$, where $u_{11} \neq 0$ and $\gamma_{1}=\frac{1}{p}>0$.

Also, let $\boldsymbol{\Psi}_{\mathbf{1}}=\left(\psi_{11}, \psi_{12}, \psi_{13}\right)^{T}$ represents the eigenvectors of $J_{1}{ }^{T}$ that of $\lambda_{11}^{*}=0$. Then again, simple calculation shows that $\boldsymbol{\Psi}_{\mathbf{1}}=\left(\psi_{11}, \gamma_{2} \psi_{11}, 0\right)^{T}$, where $\psi_{11} \neq 0$ and $\gamma_{2}=$ $\frac{\alpha p}{\left(\alpha+d_{1}\right) p+a_{2} K_{2}}>0$. 
Since the partial derivative of vector field $\boldsymbol{F}$ w.r.t the parameter $r_{1}$ is given by $\frac{\partial \boldsymbol{F}}{\partial r_{1}}=$ $\left(S\left(1-\frac{S+I}{K_{1}}\right), 0,0\right)^{t}$, hence, by substituting $P_{2}$ and $r_{1}{ }^{*}$ in this derivative, we obtain that $\boldsymbol{F}_{r_{1}}\left(P_{2}, r_{1}^{*}\right)=(0,0,0)^{t}$. Therefore, $\boldsymbol{\Psi}_{1}{ }^{t}\left[\boldsymbol{F}_{r_{1}}\left(P_{2}, r_{1}^{*}\right)\right]=0$.

Thus the system (1) at $P_{2}$ with $r_{1}=r_{1}{ }^{*}$ does not experience SNB in view of Sotomayor theorem. Moreover, since

$$
\boldsymbol{\Psi}_{\mathbf{1}}{ }^{t}\left[D \boldsymbol{F}_{r_{1}}\left(P_{2}, r_{1}{ }^{*}\right) \boldsymbol{U}_{\mathbf{1}}\right]=u_{11} \psi_{11} \neq 0
$$

where $D \boldsymbol{F}_{r_{1}}$ represents the derivative of $\boldsymbol{F}_{r_{1}}$ w.r.t $\boldsymbol{X}$,

then

$\boldsymbol{\Psi}_{\mathbf{1}}{ }^{t}\left[D^{2} \boldsymbol{F}\left(P_{2}, r_{1}{ }^{*}\right)\left(\boldsymbol{U}_{\mathbf{1}}, \boldsymbol{U}_{\mathbf{1}}\right)\right]=-2 a_{1} \psi_{11} u_{11}{ }^{2}\left(\frac{K_{2}}{p K_{1}}+\gamma_{1}\right) \neq 0$,

where $D^{2} \boldsymbol{F}$ represents the second derivative of $\boldsymbol{F}$ w.r.t. $\boldsymbol{X}$ that is given by equation (22). Accordingly, by Sotomayor theorem [26], the system (1) near the EP, $P_{2}$, with $r_{1}=r_{1}{ }^{*}$ possesses a TB but not PB.

Theorem 9. Assume that the conditions (11b)-(11d) hold and the parameter $\varepsilon$ satisfies

$$
\varepsilon \equiv \varepsilon^{*}=\frac{r_{2}}{a_{2} \vec{I}}
$$

Then, the system (1) near the predator-free EP, $P_{3}$, has a TB provided that the following condition holds, otherwise it has a PB:

$$
\frac{\beta_{2}}{\bar{I}}+\frac{p}{\left(K_{2}+\bar{S}+\bar{I}\right)} \neq 0,
$$

Proof: Note that, when $\varepsilon=\varepsilon^{*}$, then the JM of system (1) at $P_{3}$ can be written as

$J_{2}=J\left(P_{3}, \varepsilon^{*}\right)=\left[\begin{array}{ccc}a_{11} & a_{12} & a_{13} \\ a_{21} & a_{22} & a_{23} \\ 0 & 0 & 0\end{array}\right]$,

where $a_{i j} ; i=1,2 ; j=1,2,3$ are given in equation (10a). Clearly, $J_{2}$ has zero eigenvalue $\lambda_{23}{ }^{*}=0$ with two other eigenvalues, that are given by Eq. (10c), having negative real parts due to conditions (11b)-(11d).

Let $\boldsymbol{U}_{2}=\left(u_{21}, u_{22}, u_{23}\right)^{t}$ be the eigenvectors of $J_{2}$ of $\lambda_{23}{ }^{*}=0$.

So, direct computation shows that $\boldsymbol{U}_{2}=\left(\beta_{1} u_{23}, \beta_{2} u_{23}, u_{23}\right)^{t}$, where $u_{23} \neq 0$ (any real number) and $\beta_{1}=\frac{a_{12} a_{23}-a_{22} a_{13}}{a_{11} a_{22}-a_{12} a_{21}}$ and $\beta_{2}=\frac{a_{23} a_{11}-a_{21} a_{13}}{a_{11} a_{22}-a_{12} a_{21}}<0$.

Let $\boldsymbol{\Psi}_{2}=\left(\psi_{21}, \psi_{22}, \psi_{23}\right)^{t}$ represents the eigenvectors of $J_{2}{ }^{t}$ that $\lambda_{23}{ }^{*}=0$. Then, a straightforward calculation shows that $\Psi_{2}=\left(0,0, \psi_{23}\right)^{t}$, where $\psi_{23} \neq 0$ (any real number).

Since $\frac{\partial \boldsymbol{F}}{\partial \varepsilon}=\left(0,0,-a_{2} I Y\right)^{t}$, hence we obtain that $\boldsymbol{F}_{\varepsilon}\left(P_{3}, \varepsilon^{*}\right)=(0,0,0)^{T}$.

Therefore, $\boldsymbol{\Psi}_{2}{ }^{t}\left[\boldsymbol{F}_{\varepsilon}\left(P_{3}, \varepsilon^{*}\right)\right]=0$. Thus, system (1) at EP $P_{3}$ with $\varepsilon=\varepsilon^{*}$ does not undergo

SNB in view of Sotomayor theorem.

Now, since

$\boldsymbol{\Psi}_{2}{ }^{t}\left[D \boldsymbol{F}_{\varepsilon}\left(P_{3}, \varepsilon^{*}\right) \boldsymbol{U}_{2}\right]=-a_{2} \bar{I} u_{23} \psi_{23} \neq 0$,

and

$\boldsymbol{\Psi}_{2}{ }^{t}\left[D^{2} \boldsymbol{F}\left(P_{3}, \varepsilon^{*}\right)\left(\boldsymbol{U}_{2}, \boldsymbol{U}_{2}\right)\right]=-2 r_{2} \psi_{23} u_{23}{ }^{2}\left[\frac{\beta_{2}}{\bar{I}}+\frac{p}{\left(K_{2}+\bar{S}+\bar{I}\right)}\right]$,

then clearly, $\boldsymbol{\Psi}_{2}{ }^{t}\left[D^{2} \boldsymbol{F}\left(P_{3}, \varepsilon^{*}\right)\left(\boldsymbol{U}_{2}, \boldsymbol{U}_{2}\right)\right] \neq 0$ due to condition (26) and hence the system (1) undergoes a TB near $P_{2}$ when $\varepsilon=\varepsilon^{*}$. However, violating condition (26) leads to $\boldsymbol{\Psi}_{\mathbf{2}}{ }^{t}\left[D^{2} \boldsymbol{F}\left(P_{3}, \varepsilon^{*}\right)\left(\boldsymbol{U}_{2}, \boldsymbol{U}_{2}\right)\right]=0$. Furthermore, using equation (23) gives

$\boldsymbol{\Psi}_{2}{ }^{t}\left[D^{3} \boldsymbol{F}\left(P_{2}, \alpha^{*}\right)\left(\boldsymbol{V}_{2}, \boldsymbol{V}_{2}, \boldsymbol{V}_{2}\right)\right] \neq 0$.

Hence, system (1) undergoes PB.

Theorem 10. Assume that the condition (13b) holds and the parameter $d_{1}$ satisfies that

$$
d_{1} \equiv d_{1}{ }^{*}=b_{1} \tilde{S}-\alpha-a_{2} \tilde{Y} \text {. }
$$


Then, system (1) near the infected prey-free EP, $P_{4}$, has a TB, provided that the following condition holds, otherwise it has a PB:

$$
b_{1} \rho_{1}-a_{2} \rho_{2}-\frac{n \tilde{s}}{m} \neq 0
$$

Proof: Note that, when $d_{1}=d_{1}{ }^{*}$, then the JM of the system (1) at $P_{4}$ can be written as

$$
J_{3}=J\left(P_{4}, d_{1}^{*}\right)=\left[\begin{array}{ccc}
r_{1}-\frac{2 r_{1} \tilde{S}}{K_{1}}-a_{1} \tilde{Y} & -\frac{r_{1} \tilde{S}}{K_{1}}-b_{1} \tilde{S}+\alpha & -a_{1} \tilde{S} \\
0 & 0 & 0 \\
\frac{r_{2}}{p} & \frac{r_{2}}{p}-\varepsilon a_{2} \tilde{Y}^{2} & -r_{2}
\end{array}\right]=\left(m_{i j}\right) .
$$

Clearly, $J_{3}$ has $\lambda_{32}{ }^{*}=0$ with two other eigenvalues, given by Eq. (12b), having negative real parts due to condition (13b).

Clearly, the necessary but not sufficient condition for bifurcation is satisfied and $P_{4}$ is a nonhyperbolic point.

Let $\boldsymbol{U}_{3}=\left(u_{31}, u_{32}, u_{33}\right)^{t}$ be the eigenvectors of $J_{3}$ of $\lambda_{32}^{*}=0$. Then, simple computation gives that $\boldsymbol{U}_{\mathbf{3}}=\left(\rho_{1} u_{32}, u_{32}, \rho_{2} u_{32}\right)^{t}$, where $u_{32} \neq 0, \rho_{1}=\frac{m_{32} m_{13}-m_{12} m_{11}}{m_{11} m_{33}-m_{13} m_{31}}$, and $\rho_{2}=$ $\frac{m_{12} m_{31}-m_{32} m_{11}}{m_{11} m_{33}-m_{13} m_{31}}$.

Also, let $\boldsymbol{\Psi}_{3}=\left(\psi_{31}, \psi_{32}, \psi_{33}\right)^{t}$ represents the eigenvectors of $J_{3}{ }^{t}$ that of $\lambda_{32}^{*}=0$. Then again, simple calculation shows that $\boldsymbol{\Psi}_{3}=\left(0, \psi_{32}, 0\right)^{t}$, where $\psi_{11} \neq 0$.

Since the partial derivative of vector field $\boldsymbol{F}$ w.r.t the parameter $d_{1}$ is given by $\frac{\partial \boldsymbol{F}}{\partial d_{1}}=$ $(0,-I, 0)^{t}$, hence by substituting $P_{4}$ and $d_{1}{ }^{*}$ in this derivative we obtain that $\boldsymbol{F}_{d_{1}}\left(P_{4}, d_{1}{ }^{*}\right)=$ $(0,0,0)^{t}$. Therefore, $\boldsymbol{\Psi}_{3}{ }^{t}\left[\boldsymbol{F}_{d_{1}}\left(P_{4}, d_{1}{ }^{*}\right)\right]=0$.

Thus, the system (1) at $P_{4}$ with $d_{1}=d_{1}{ }^{*}$ does not experience SNB in view of Sotomayor theorem. Moreover, since

then,

$$
\boldsymbol{\Psi}_{3}{ }^{t}\left[D \boldsymbol{F}_{d_{1}}\left(P_{4}, d_{1}^{*}\right) \boldsymbol{U}_{3}\right]=-u_{32} \psi_{32} \neq 0,
$$

$\boldsymbol{\Psi}_{\mathbf{3}}{ }^{t}\left[D^{2} \boldsymbol{F}\left(P_{4}, d_{1}{ }^{*}\right)\left(\boldsymbol{U}_{\mathbf{3}}, \boldsymbol{U}_{3}\right)\right]=2 \psi_{32} u_{32}{ }^{2}\left(b_{1} \rho_{1}-a_{2} \rho_{2}-\frac{n \tilde{s}}{m}\right)$.

Clearly, $\boldsymbol{\Psi}_{3}{ }^{t}\left[D^{2} \boldsymbol{F}\left(P_{4}, d_{1}{ }^{*}\right)\left(U_{3}, \boldsymbol{U}_{3}\right)\right] \neq 0$ due to condition (27b) and hence the system (1) undergoes a TB near $P_{4}$ when $d_{1}=d_{1}{ }^{*}$. However, violating condition (27b) leads to $\boldsymbol{\Psi}_{3}{ }^{t}\left[D^{2} \boldsymbol{F}\left(P_{4}, d_{1}{ }^{*}\right)\left(U_{3}, \boldsymbol{U}_{3}\right)\right]=0$. Furthermore, using equation (23) gives

$\boldsymbol{\Psi}_{\mathbf{2}}{ }^{t}\left[D^{3} \boldsymbol{F}\left(P_{2}, \alpha^{*}\right)\left(\boldsymbol{V}_{\mathbf{2}}, \boldsymbol{V}_{\mathbf{2}}, \boldsymbol{V}_{\mathbf{2}}\right)\right]=-2 \frac{n}{m} \psi_{32} u_{32}{ }^{3}\left(3 \rho_{1}+\frac{2 \tilde{s}}{m^{2}}\right) \neq 0$

Hence, system (1) undergoes PB.

Theorem 11. Assume the conditions (15a)-(15b) along with the

$$
\begin{gathered}
\frac{2 r_{2} p Y^{*}}{\left(K_{2}+S^{*}+I^{*}\right)}+\varepsilon a_{2} I^{*}<r_{2}, \\
2 \Gamma_{11}<\Gamma_{10} .
\end{gathered}
$$

Then, as the parameter $a_{1}$ passes through the value

$$
a_{1}{ }^{*}=\frac{1}{S^{*}}\left[\frac{e_{33}\left(e_{11} e_{22}-e_{12} e_{21}\right)+e_{23}\left(e_{12} e_{31}-e_{11} e_{32}\right)}{\left(e_{21} e_{32}-e_{22} e_{31}\right)}\right] \text {, }
$$

Then, the system (1) near the coexistence EP, $P_{5}$, has a SNB provided that

$$
\xi_{1}{ }^{2} q_{11}{ }^{*}+\left(\xi_{3}+\xi_{4}\right) q_{21}{ }^{*}+q_{31}{ }^{*}+q_{41}{ }^{*} \neq 0,
$$

where all the symbols are given in the proof.

Proof: Straightforward computation shows that under the conditions (15a), (15b), (27a), and (28b) with $a_{1}=a_{1}{ }^{*}$, the coefficients of the characteristic equation given by Eq. (14b) are $M_{1}>0, M_{2}>0$ and $M_{3}=0$. Hence, Eq. (14b) has three roots (eigenvalues of $J\left(P_{5}\right)$ ) given by

$\lambda_{41}{ }^{*}=0, \lambda_{42}{ }^{*}=-\frac{M_{1}}{2}+\frac{1}{2} \sqrt{M_{1}{ }^{2}-4 M_{2}}, \lambda_{43}{ }^{*}=-\frac{M_{1}}{2}-\frac{1}{2} \sqrt{M_{1}{ }^{2}-4 M_{2}}$,

where $M_{1}, M_{2}$ and $M_{3}$ are defined in equation (14b). 
Clearly, the eigenvalues $\lambda_{42}{ }^{*}$ and $\lambda_{43}{ }^{*}$ have negative real parts due to conditions (15a) and (15b). Hence, the Jacobian matrix of the system (1) of $P_{5}$ and $a_{1}=a_{1}{ }^{*}$ can be written as $J_{4}=J\left(P_{5}, a_{1}{ }^{*}\right)=\left(e_{i j}{ }^{*}\right)_{3 \times 3}$ with $e_{i j}{ }^{*}=e_{i j} ; \forall i, j$ with $e_{11}{ }^{*}=e_{11}\left(a_{1}{ }^{*}\right)$, and $e_{13}{ }^{*}=e_{13}\left(a_{1}{ }^{*}\right)$, where $e_{i j}$ are given in Eq. (14a). We will drop the star for simplification.

Let $\boldsymbol{U}_{4}=\left(u_{41}, u_{42}, u_{43}\right)^{t}$ be the eigenvector of $J_{4}$ corresponding to $\lambda_{41}{ }^{*}=0$. Then, direct computation shows that $\boldsymbol{U}_{4}=\left(\xi_{1} u_{43}, \xi_{2} u_{43}, u_{43}\right)^{t}$, where $u_{43} \neq 0, \xi_{1}=\frac{e_{12} e_{23}-e_{13} e_{22}}{e_{11} e_{22}-e_{12} e_{21}}$, and $\xi_{2}=\frac{e_{13} e_{21}-e_{23} e_{11}}{e_{11} e_{22}-e_{12} e_{21}}$.

Let $\boldsymbol{\Psi}_{\mathbf{4}}=\left(\psi_{41}, \psi_{42}, \psi_{43}\right)^{t}$ represents the eigenvector of $J_{4}{ }^{t}$ that of $\lambda_{41}{ }^{*}=0$. Then, simple calculation shows that $\Psi_{4}=\left(\xi_{3} \psi_{43}, \xi_{4} \psi_{43}, \psi_{43}\right)^{t}$, where $\psi_{43} \neq 0, \xi_{3}=\frac{e_{12} e_{32}-e_{22} e_{31}}{a_{11} a_{22}-a_{12} a_{21}}$, and $\xi_{4}=\frac{e_{12} e_{31}-e_{32} e_{11}}{a_{11} a_{22}-a_{12} a_{21}}$.

We have that $\frac{\partial \boldsymbol{F}}{\partial a_{1}}=(-S Y, 0,0)^{t}$, hence we obtain that $\boldsymbol{F}_{a_{1}}\left(P_{5}, a_{1}^{*}\right)=\left(-S^{*} Y^{*}, 0,0\right)^{t}$.

Therefore, we obtain that

$\boldsymbol{\Psi}_{4}{ }^{t}\left[\boldsymbol{F}_{a_{1}}\left(P_{5}, a_{1}{ }^{*}\right)\right]=-\xi_{3} \psi_{43} s^{*} Y^{*} \neq 0$.

Consequently, the first condition of SNB in view of Sotomayor theorem is satisfied. Now, since:

$\boldsymbol{\Psi}_{\mathbf{4}}{ }^{t}\left[D^{2} \boldsymbol{F}\left(P_{5}, a_{1}{ }^{*}\right)\left(\boldsymbol{U}_{\mathbf{4}}, \boldsymbol{U}_{\mathbf{4}}\right)\right]=u_{43}{ }^{2} \psi_{43}\left[\xi_{1}{ }^{2} q_{11}{ }^{*}+\left(\xi_{3}+\xi_{4}\right) q_{21}{ }^{*}+q_{31}{ }^{*}+q_{41}{ }^{*}\right]$,

where $q_{11}{ }^{*}=\frac{2 r_{1}}{K_{1}} \xi_{3}, q_{21}{ }^{*}=\left[\left(2 b_{1}-\frac{4 n I^{*}}{m+I^{*}}+\frac{2 n I^{* 2}}{\left(m+I^{*}\right)^{2}}\right) \xi_{1} \xi_{2}+\left(\frac{2 n m s^{*}}{\left(m+I^{*}\right)^{2}}-\frac{2 n m S^{*}}{\left(m+I^{*}\right)^{3}}\right)_{2}{ }^{2}\right]$,

$q_{31}{ }^{*}=-2\left[\left(\frac{r_{1}}{K_{1}}+a_{1} \xi_{2}\right) \xi_{1} \xi_{3}+a_{2} \xi_{2}\left(\xi_{4}+\varepsilon\right)\right]$,

$q_{41}^{*}=\frac{-2 r_{2} p}{\left(K_{2}+S^{*}+I^{*}\right)}\left[\frac{Y^{* 2}}{\left(K_{2}+S^{*}+I^{*}\right)}\left(\xi_{1}+\xi_{2}\right)^{2}+\frac{2 Y}{\left(K_{2}+S^{*}+I^{*}\right)}\left(\xi_{1}+1\right)-1\right]$.

Clearly, $\boldsymbol{\Psi}_{4}{ }^{T}\left[D^{2} \boldsymbol{F}\left(P_{5}, a_{1}{ }^{*}\right)\left(\boldsymbol{U}_{4}, \boldsymbol{U}_{4}\right)\right] \neq 0$ under the condition (28b), and hence the system (1) undergoes SNB near the coexistence equilibrium.

\section{Numerical Simulation}

In this section, the global dynamics of the system (1) is further investigated. To specify the control set of parameters, the system is solved numerically using Runge-Kutta of ordered six, followed by forth steps Predictor- Corrector method. Then, all the obtained numerical results are drawn in the form of $3 D$ phase portraits and $2 D$ time series using Matlab version 6 . Therefore, in order to run simulations, the following hypothetical set of biological data is used in this section:

$$
\begin{gathered}
r_{1}=5, K_{1}=20, r_{2}=2, K_{2}=50, a_{1}=0.3, a_{2}=0.1, \\
b_{1}=0.15, b_{2}=0.1, p=4, m=20, \varepsilon=0.1, d_{1}=0.05, \alpha=0.5
\end{gathered}
$$

It is observed, for this set of data, that the system (1) approaches asymptotically to the unique coexistence EP, $P_{5}=(8.67,2.66,6.49)$, starting from five different initial values, as shown in Figures 1 and 2. 


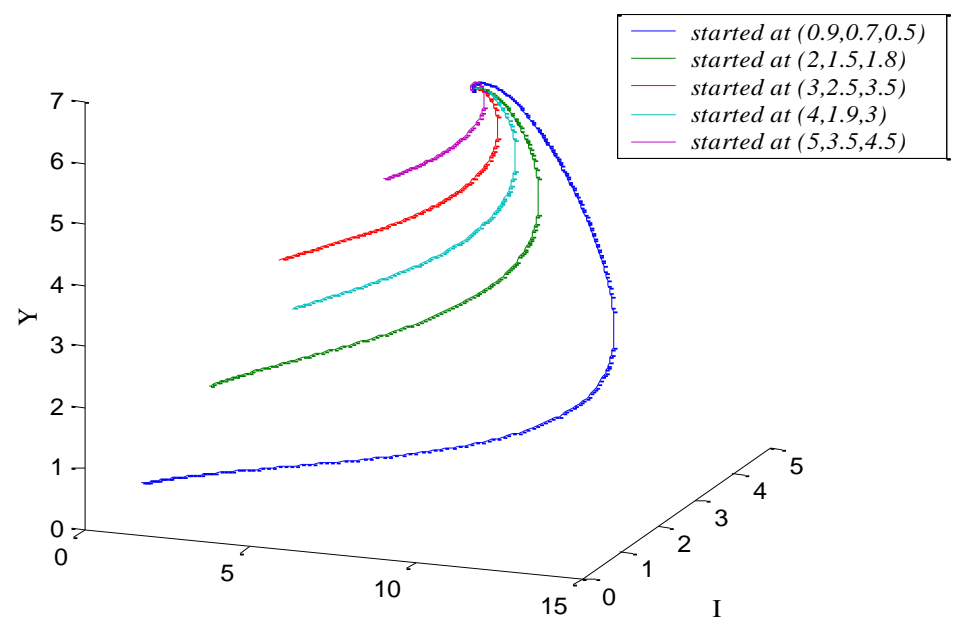

Figure 1-3D phase portrait of the system (1) using the parameters given by Eq. (30) in which the solution approaches asymptotically to the $P_{5}=(8.67,2.66,6.49)$.

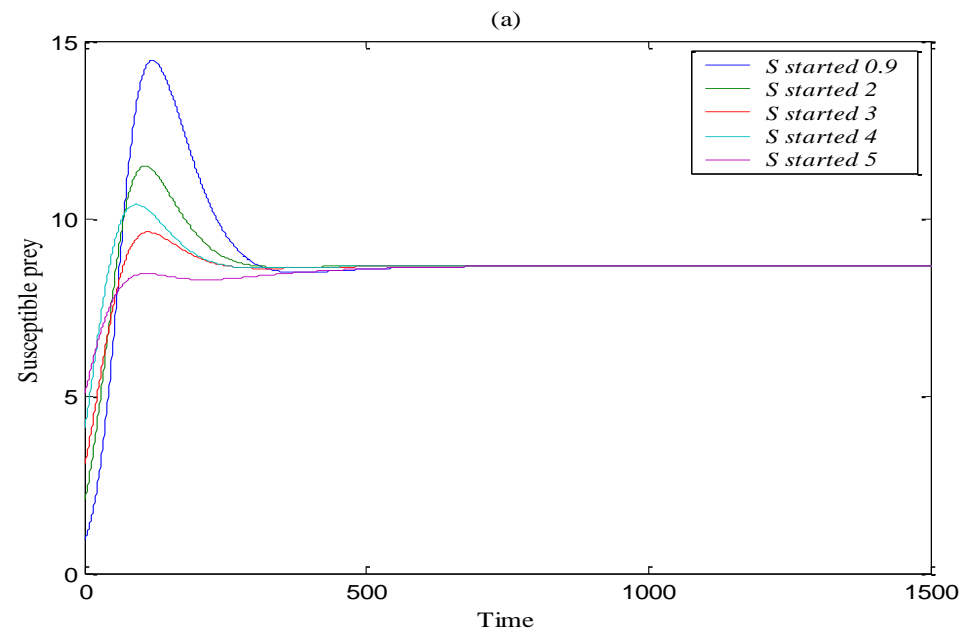

(b)

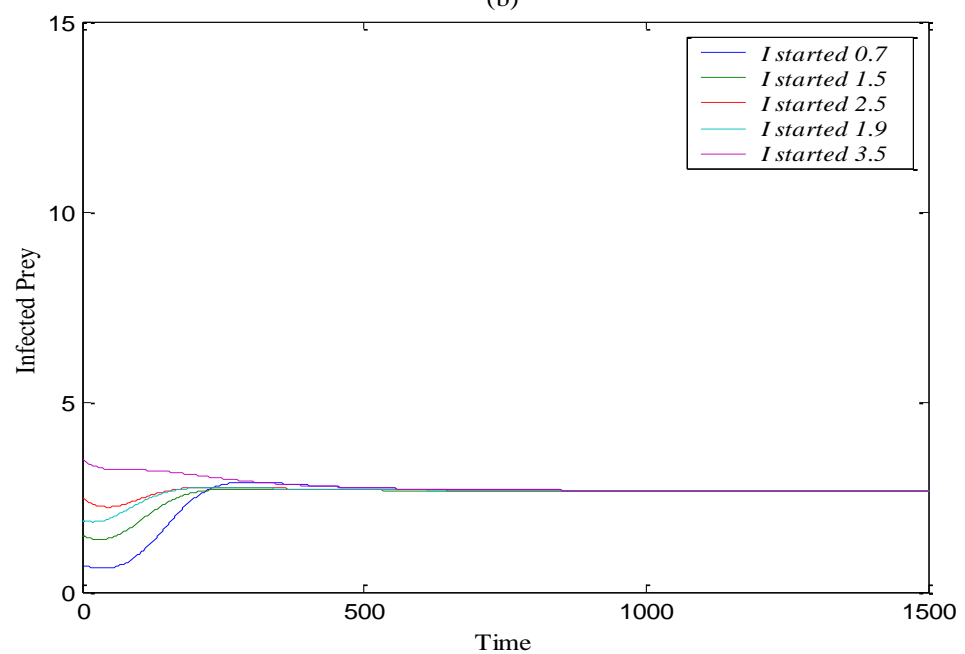




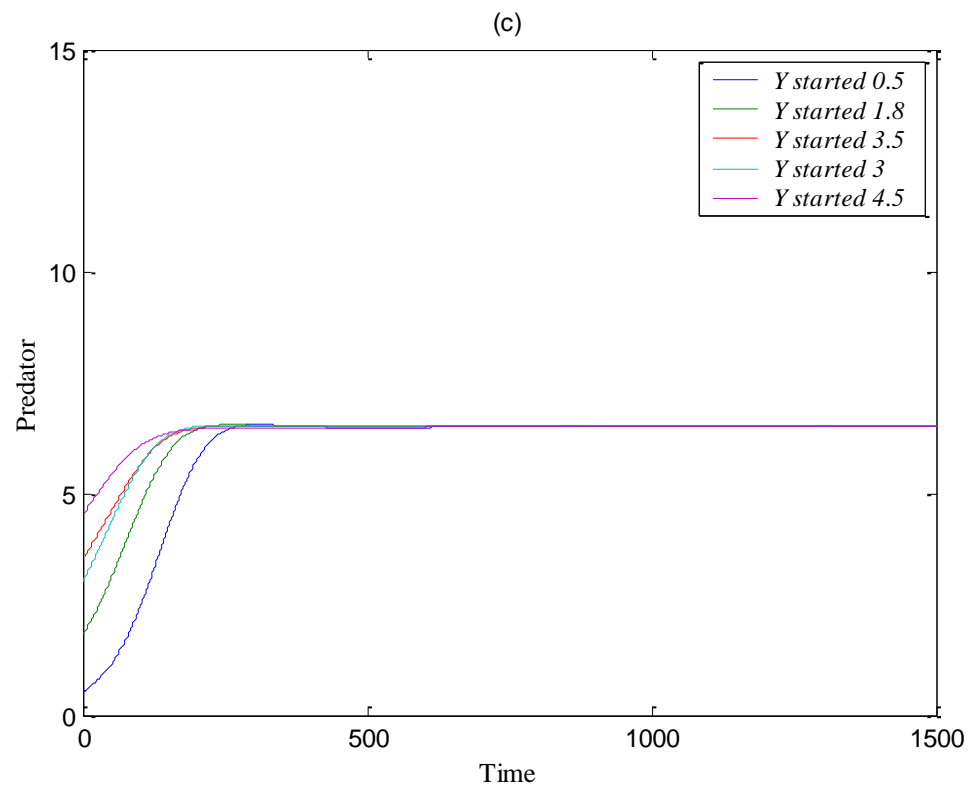

Figure 2-The solution of system (1) approaching asymptotically to $P_{5}=(8.67,2.66,6.49)$ for the data given by Eq. (30). (a) The trajectory of susceptible prey versus time. (b) The trajectory of infected prey versus time. (c) The trajectory of predator versus time.

According to these two figures, the system (1) persists at the coexistence point in $\mathbb{R}_{+}^{3}$. Now, in order to discuss the effect of varying the values of parameters on the dynamical behavior of the system, the system is solved numerically for the data given in Eq. (30), with varying a specific parameter each time and then the obtained solutions are drawn as shown below. It is observed that, for the values of parameter $r_{1}$ in the range $r_{1} \leq 2.6$ with the other parameters as in Eq. (30), the system (1) approaches asymptotically to infected prey-free EP in the interior of of $S Y$-plane; otherwise, it has a GAS coexistence EP; see Figures $3 \mathrm{a}$ and $3 \mathrm{~b}$ for typical values of $r_{1}$.

(a)

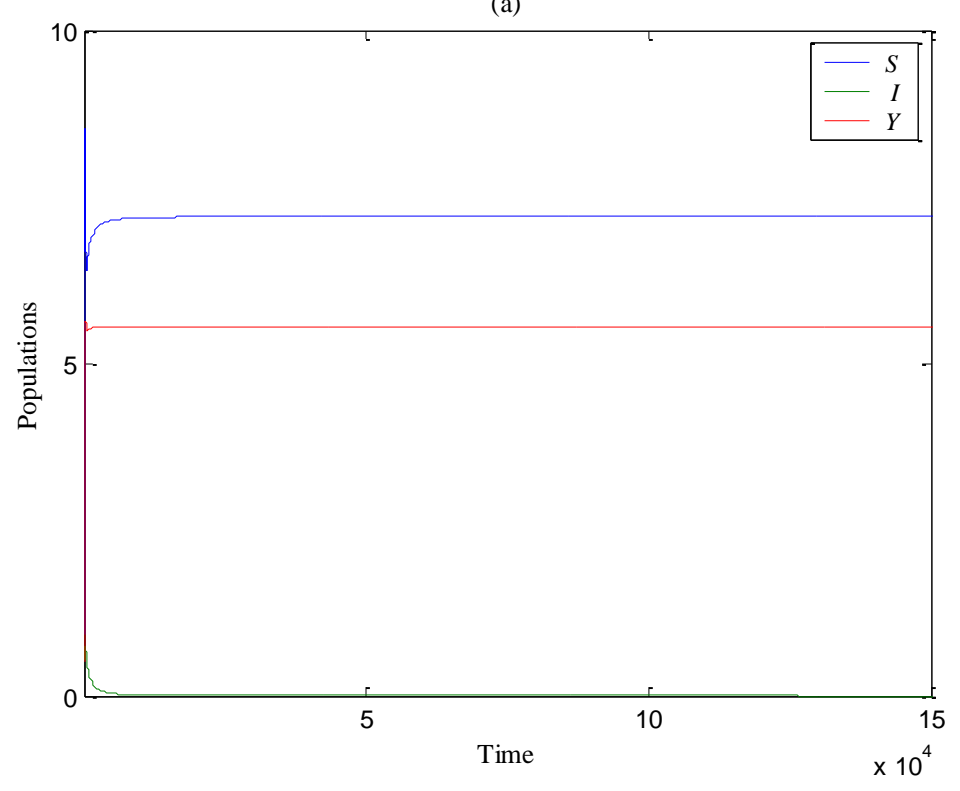


(b)

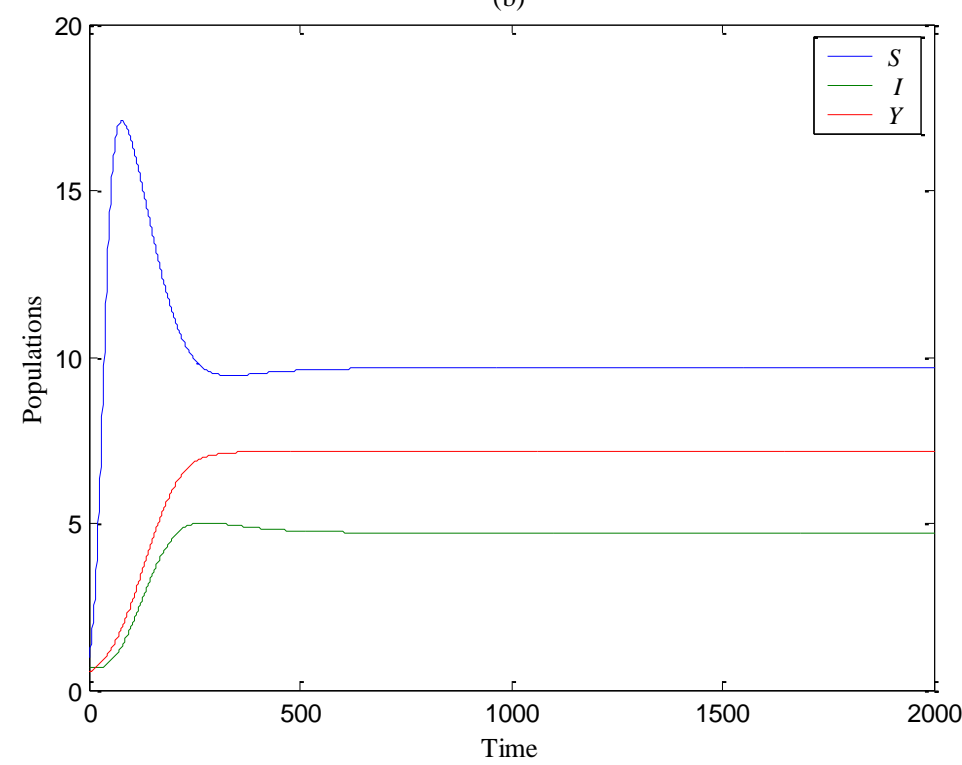

Figure 3-The trajectories of system (1) versus time for the data given by Eq. (30) with different values of $r_{1}$. (a) The system approaches asymptotically to $P_{4}=(7.19,0,5.54)$ when $r_{1}=2.6$. (b)The system approaches asymptotically to $P_{5}=(8.67,2.66,6.49)$ when $r_{1}=5$.

It is observed that varying the parameters $K_{1}$ and $b_{1}$ has a similar effect to that shown with varying $r_{1}$. Now, for the parameter $r_{2}$ in the range $r_{2} \leq 0.1$, it is observed that the system (1) approaches asymptotically to predator-free EP in the interior of $S I$-plane, as shown in the below typical figures given by Figure 4 . However, it approaches to $P_{5}$ otherwise.

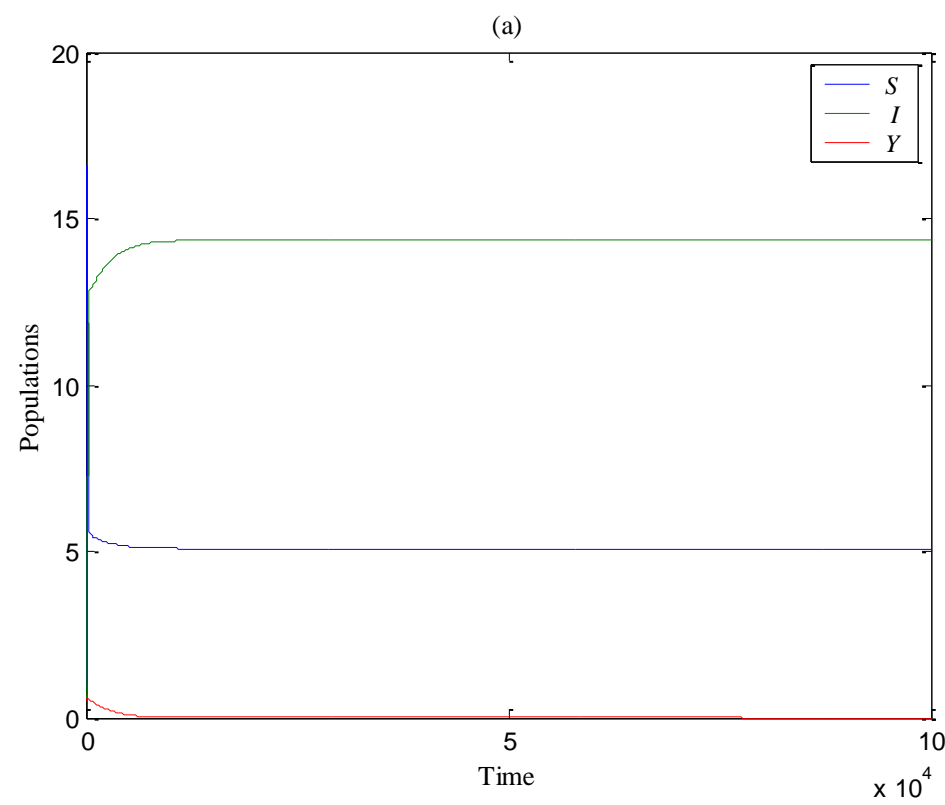


(b)

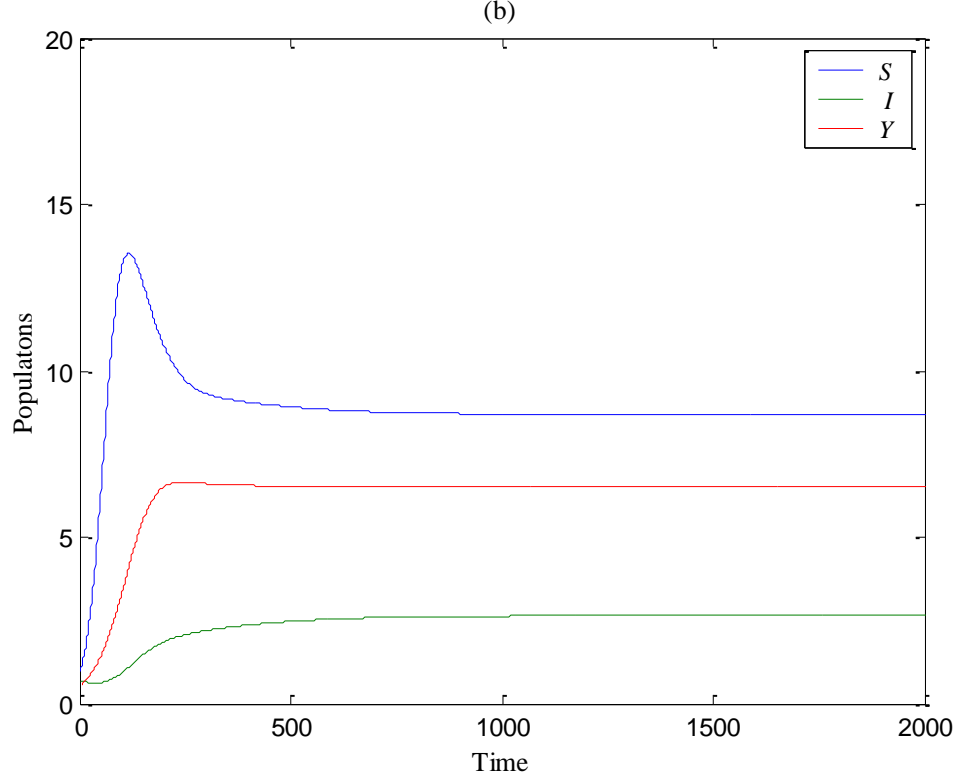

Figure 4-The trajectories of the system (1) versus time for the data given by Eq. (30) with different values of $r_{2}$. (a) The system approaches asymptotically to $P_{3}=(5.08,14.35,0)$ when $r_{2}=0.1$. (b)The system approaches asymptotically to $P_{5}=(8.68,2.63,6.52)$ when $r_{2}=2.6$. It is observed that varying the parameters $\varepsilon$ has a similar effect to that shown with varying $r_{2}$. Now, for the parameter $K_{2}$ in the range $K_{2} \leq 26$, it is observed that the system (1) approaches asymptotically to the coexistence EP, as shown typically in Figure 5. However, it approaches to $P_{4}$ otherwise.

(a)

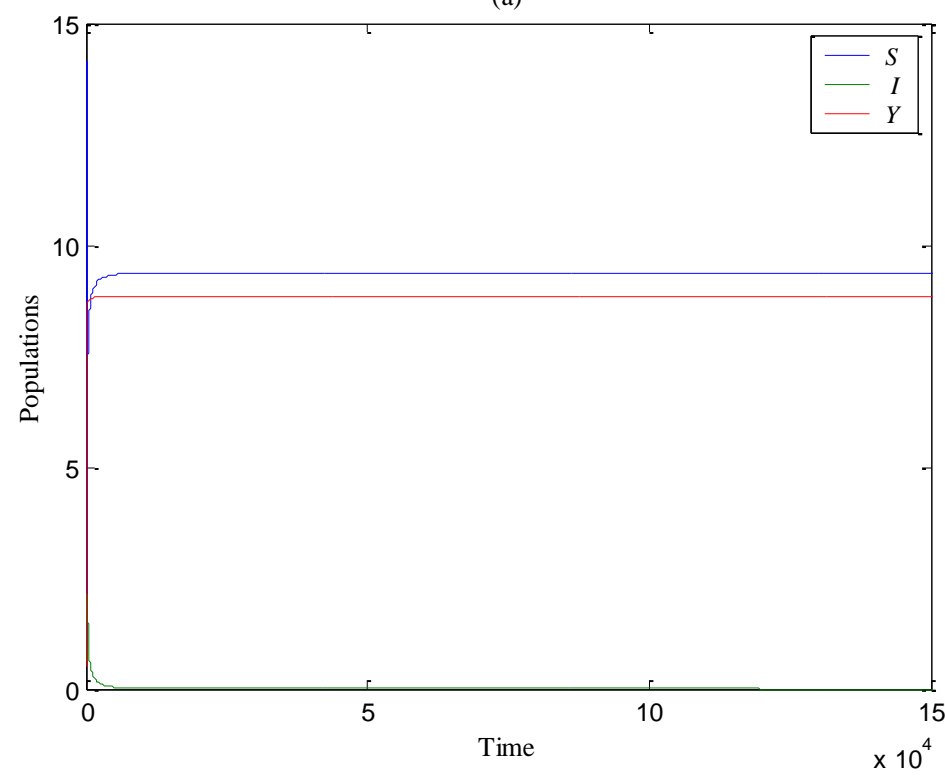


(b)

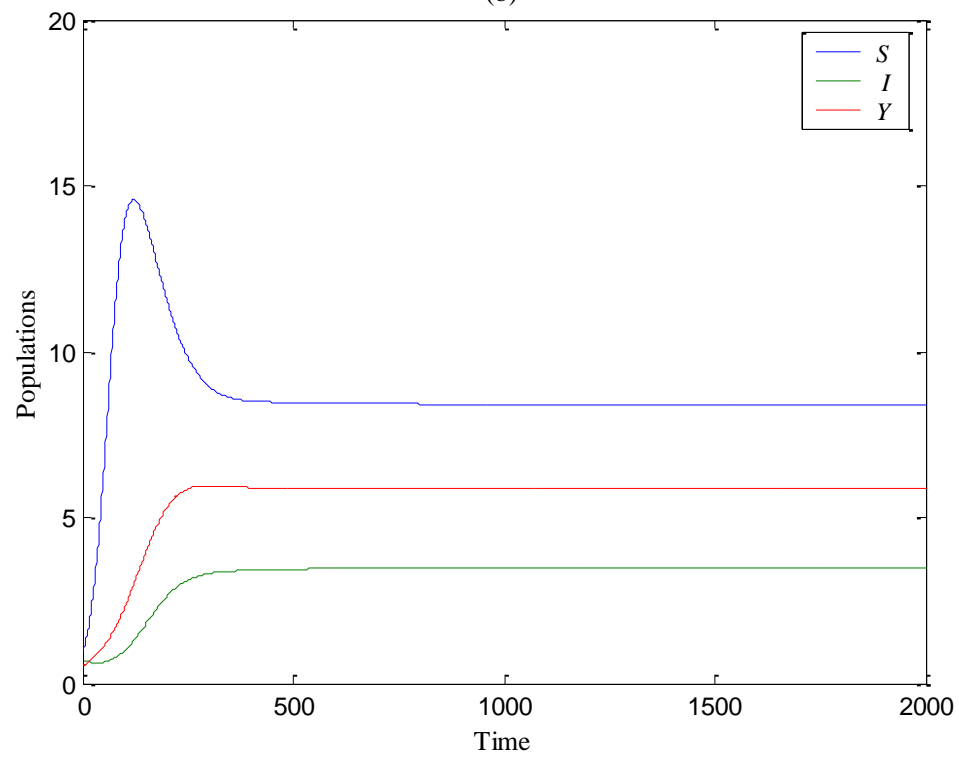

Figure 5-The trajectories of the system (1) versus time for the data given by Eq. (30) with different values of $K_{2}$. (a) The system approaches asymptotically to $\mathrm{P}_{4}=(9.38,0,8.84)$ when $\mathrm{K}_{2}=26$. (b)The system approaches asymptotically to $\mathrm{P}_{5}=(8.41,3.48,5.87)$ when $\mathrm{K}_{2}=12$.

It is observed that varying the parameters $a_{1}, \alpha$, and $d_{1}$ has similar effects as those shown with varying $K_{2}$. On the other hand, varying the parameters of the infection rate of the system (1) is also studied. It is observed that, for $b_{1} \geq 0.1$, the system (1) approaches asymptotically to the infected prey-free EP; otherwise, it has a GAS coexistence EP that has a GAS at $P_{5}$. However, for $n=0.15$ (maximum transmission rate under the media coverage alert), with increasing the response of individuals to the media coverage alert or decreasing the parameter $m$, the system (1) approaches gradually to $P_{4}$, as shown in Figure 6 for the values $m=$ $20,10,5,0$, respectively.

(a)

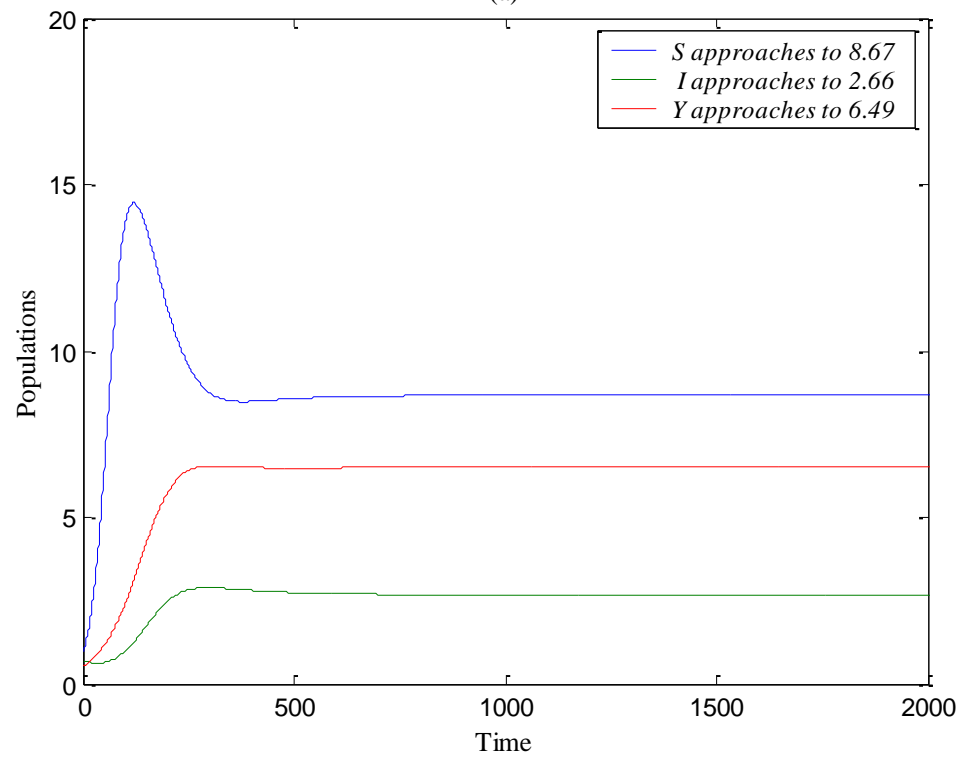


(b)

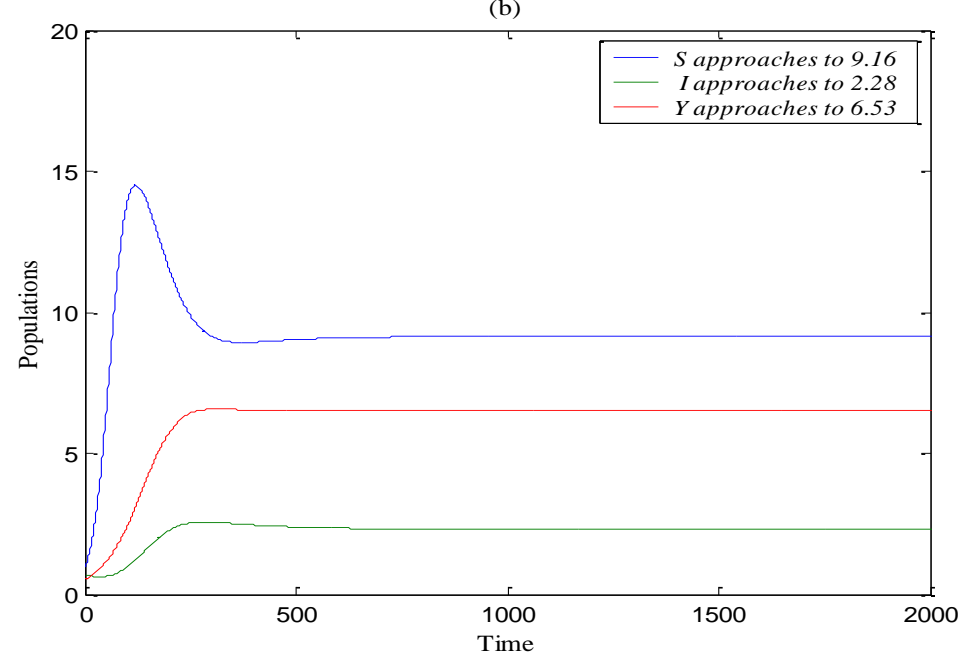

(c)

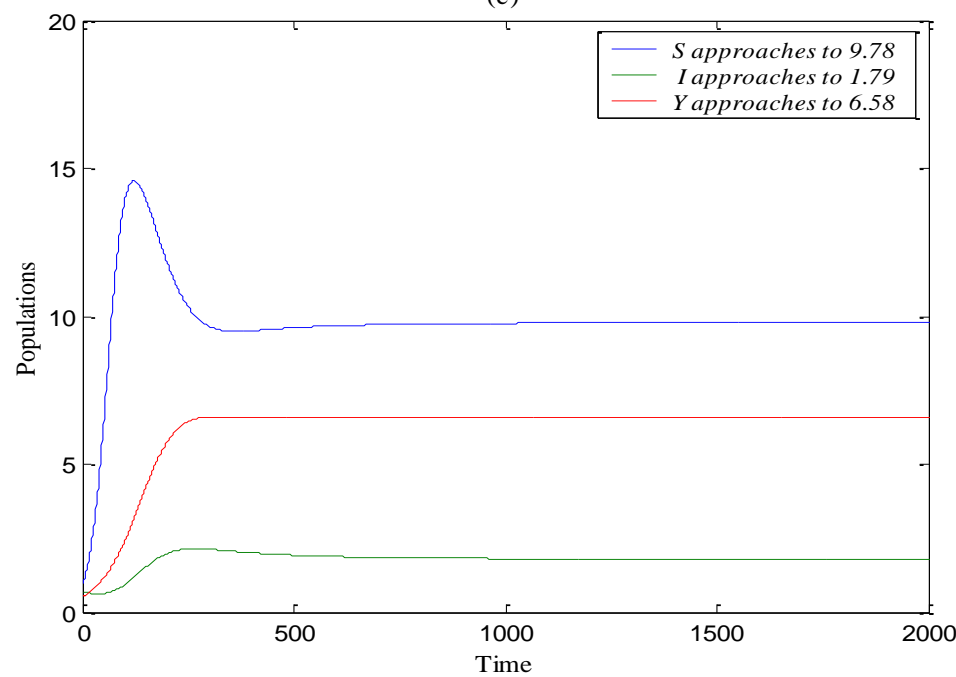

(d)

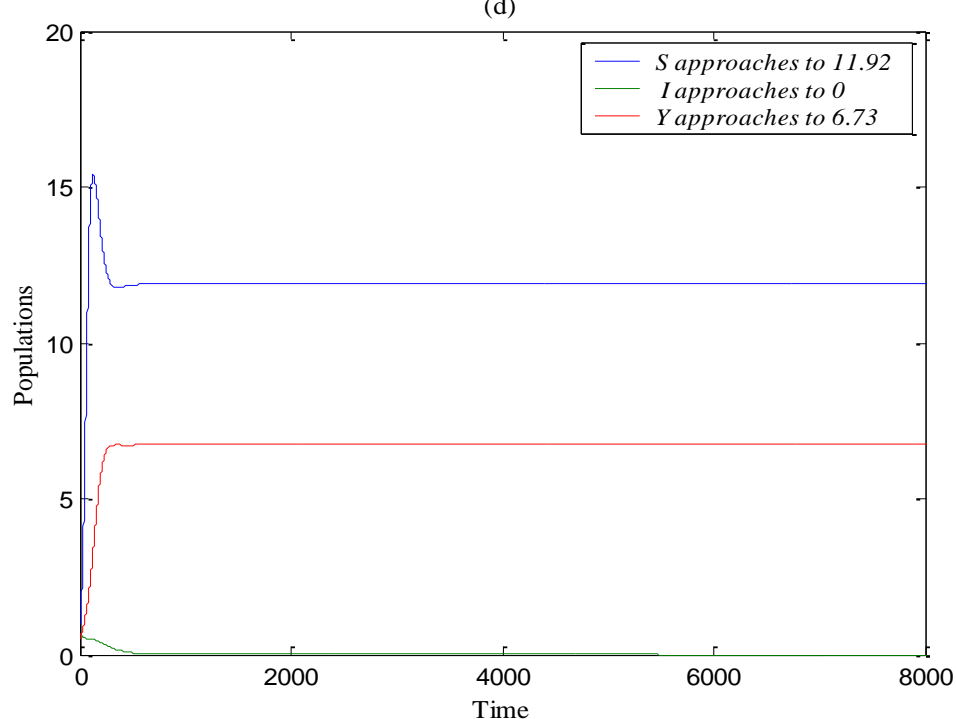

Figure 6-The trajectories of system (1) versus time for the data given by Eq. (30) with $\mathrm{n}=0.15$ and different values of $\mathrm{m}$. (a) The system approaches asymptotically to $\mathrm{P}_{5}=$ $(8.67,2.66,6.49)$ when $m=20$. (b) The system approaches asymptotically to $P_{5}=$ 
$(9.16,2.28,6.53)$ when $m=10$. (c) The system approaches asymptotically to $P_{5}=$ $(9.78,1.79,6.58)$ when $\mathrm{m}=5$. (d) The system approaches asymptotically to $\mathrm{P}_{4}=$ $(11.92,0,6.73)$ when $\mathrm{m}=0$.

Now, for the the parameter $p$ in the range $0.9 \leq p \leq 2.7$ with the rest of parameters as in Eq. (30), the system (1) approaches asymptotically to $P_{4}$. However, the system (1) approaches asymptotically to the coexistence equilibrium points $P_{5}$ and $P_{2}$ for the ranges $p>$ 2.7 and $\mathrm{p}<0.9$, respectively, as shown typically in Figure 7.

(a)

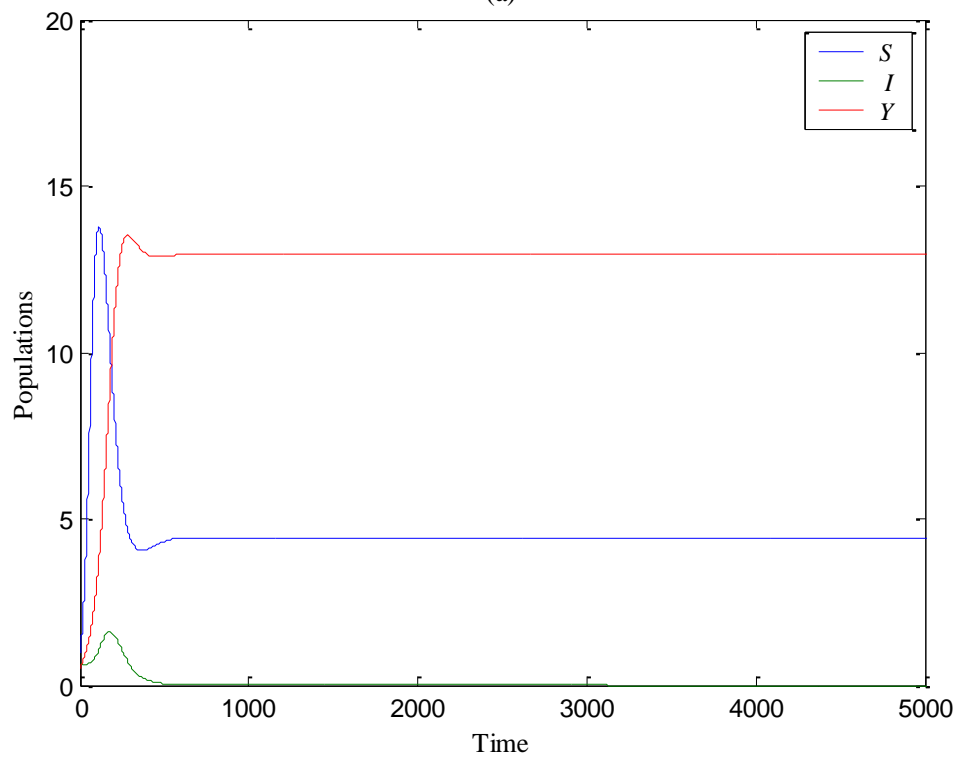

(b)

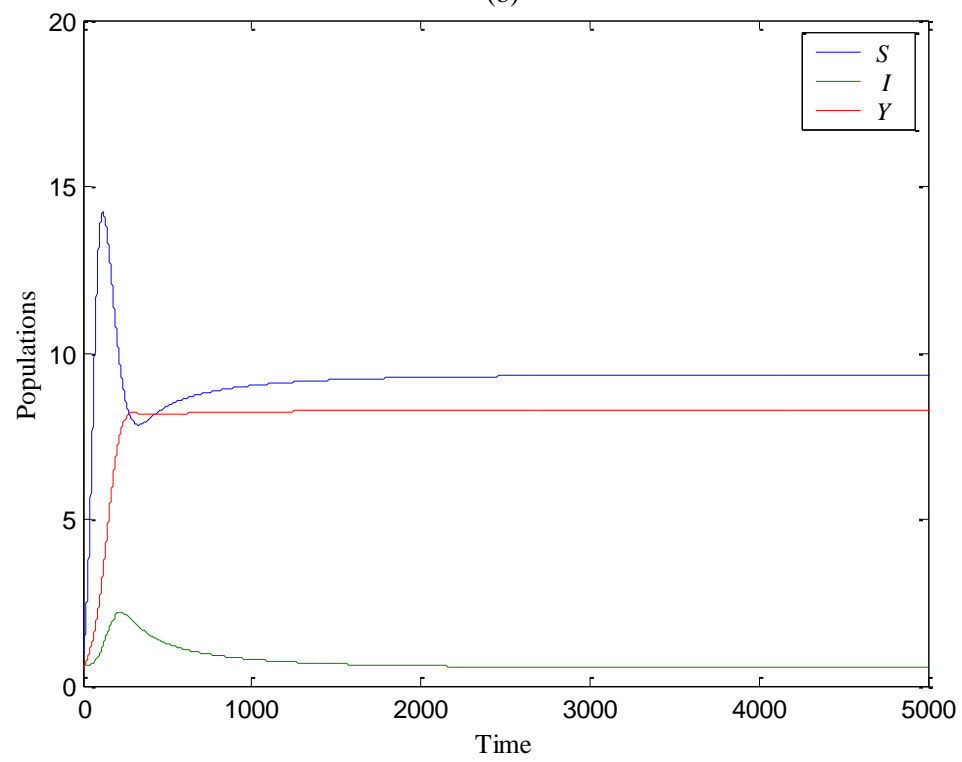


(c)

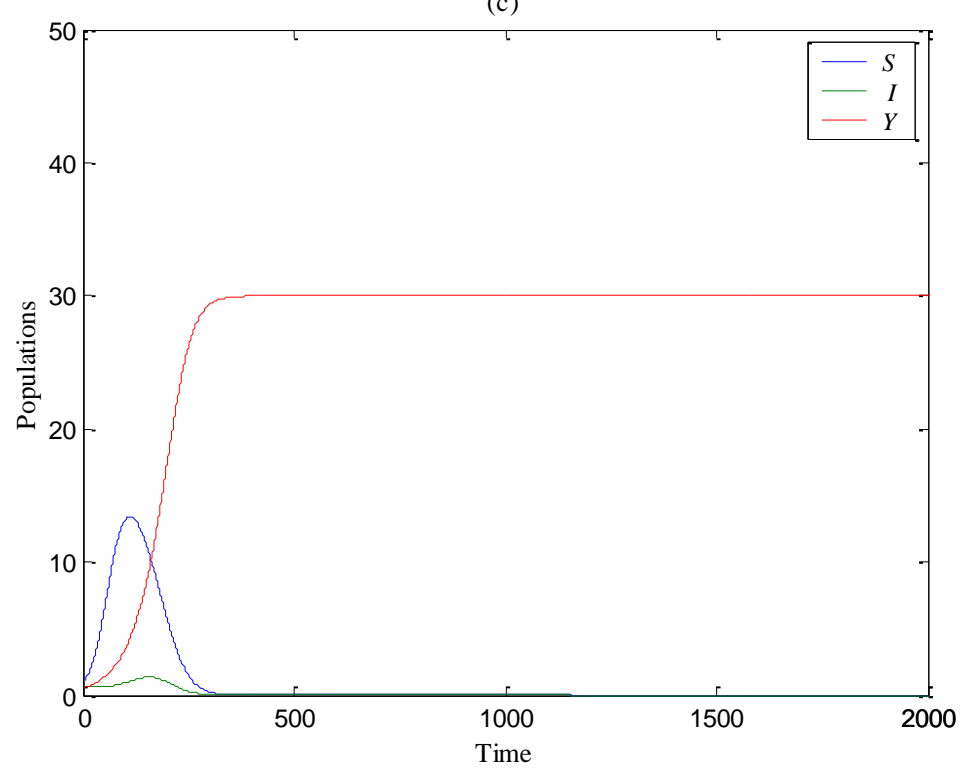

Figure 7-The trajectories of system (1) versus time for the data given by Eq.(30) with different values of $\mathrm{a}_{1}$. (a) The system approaches asymptotically to $\mathrm{P}_{4}=(4.44,0,12.96)$ when $\mathrm{p}=1.5$. (b) The system approaches asymptotically to $P_{5}=(9.39,0.53,8.26)$ when $p=3$. (c) The system approaches asymptotically to $P_{2}=(0,0,30)$ when $p=0.5$.

Finally, for the parameter $a_{2}$ in the range $0.2 \leq a_{2} \leq 2.2$, with the rest of parameters as in Eq.(30), the system (1) approaches asymptotically to $P_{4}$, as shown in Figure 8. Otherwise, the system (1) still approaches to $P_{5}$ in the interior of $\mathbb{R}_{+}^{3}$.

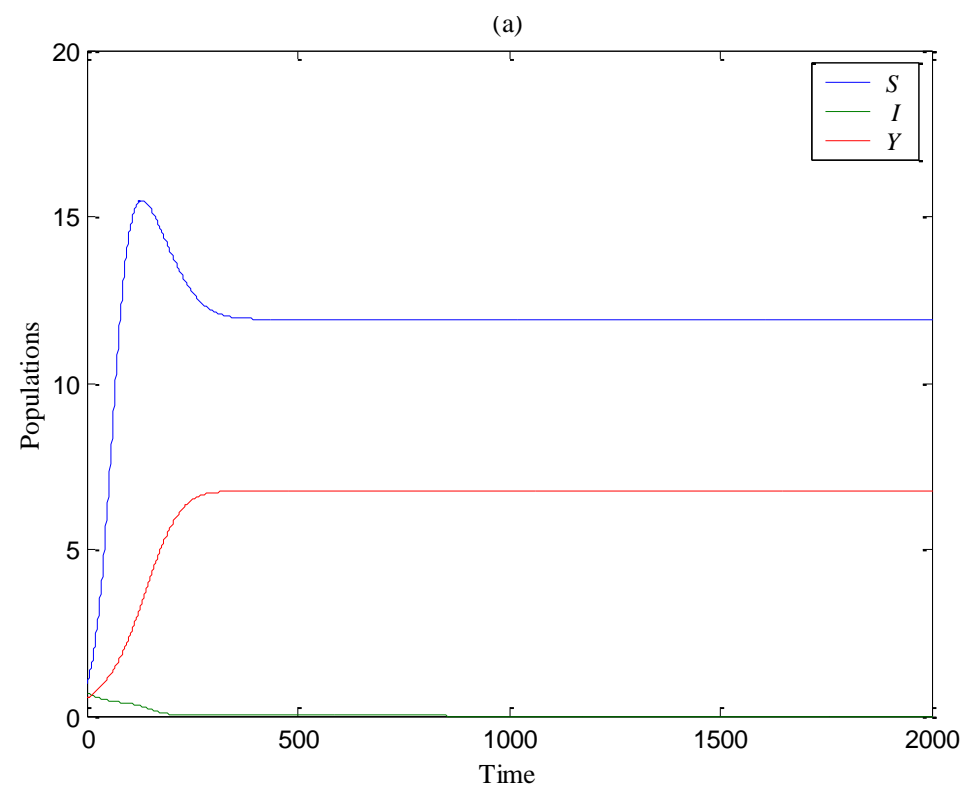


(b)

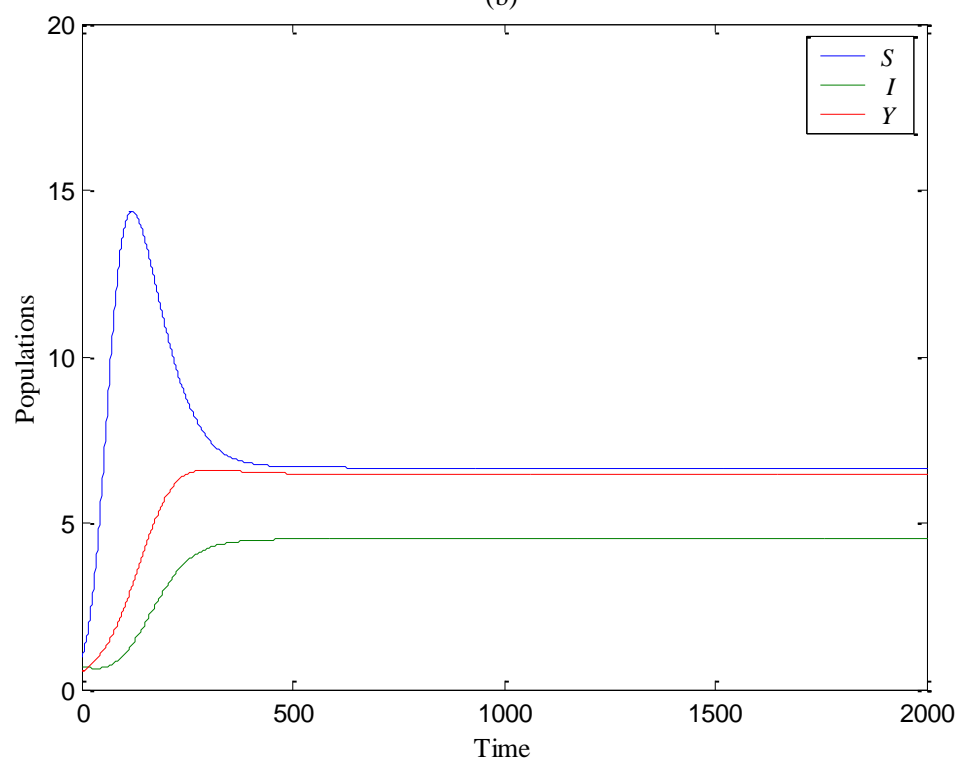

Figure 8-The trajectories of system (1) versus time for the data given by Eq. (30) with different values of $\mathrm{a}_{2}$. (a) The system approaches asymptotically to $\mathrm{P}_{4}=(11.92,0,6.73)$ when $\mathrm{a}_{2}=0.9$. (b) The system approaches asymptotically to $P_{5}=(6.64,4.55,6.47)$ when $\mathrm{a}_{2}=0.05$.

\section{Discussion and Conclusion}

In this paper, the effect of media coverage alert on the dynamical behavior of the diseased modified Leslie-Gower prey-predator model involving disease in the prey is considered. The system is studied theoretically as well as numerically. It is observed that the system has at most six non-negative equilibrium points. Since the solution of the system is proved to be uniformly bounded, it is observed that the solution approaches asymptotically to one of its equilibrium points depending on determined conditions. According to the numerical simulation, it is observed that media coverage works as a control parameter for the spread of disease.

\section{References}

[1] R. M. Anderson, R. M. May. "The invasion, persistence and spread of infectious diseases within animal and plant communities". Philos. Trans. R. Soc. London B, vol. 314, pp. 533-570, 1986.

[2] K.P. Hadeler, H.I. Freedman. "Predator-prey population with parasitic infection". J. Math. Biol., vol. 27, pp. 609-631, 1989.

[3] E. Venturino. "The influence of diseases on Lotka-Voltera systems". Rocky Mount. J. Math., vol. 24, pp. 381-402, 1984.

[4] Bairagi N., Roy P.K., and Chattopadhyay J., "Role of infection on the stability of a predator-prey system with several response functions a comparative study", Journal of Theoretical Biology, vol. 248, no. 1, pp. 10-25, 2007.

[5] Bhattacharyya R. and Mukhopadhyay, B., "On an eco-epidemiological model with prey harvesting and predator switching: local and global perspectives". Nonlinear Analysis Real World Applications, vol. 11, no. 455, pp. 3824-3833, 2010.

[6] Bob W., George, A.K. \& Krishna, D, "Stabilization and complex dynamics in a predator-prey model with predator suffering from an infectious disease", Ecol. Complex, vol. 8, pp. 113-122, 2011.

[7] Liu, X., "Bifurcation of an eco-epidemiological model with a nonlinear incidence rate". Appl. Math. Comput, vol. 218, pp. 2300-2309, 2011.

[8] A.M. Bate, F.M. Hilker, "Disease in group-defending prey can benefit predators". Theor. Ecol., vol. 7, no.1, pp. 87-100, 2014. 
[9] Biswas, S., Samanta, S. and Chattopadhyaya, J., "A model based theoretical study on cannibalistic prey-predator system with disease in both populations". Differential Equations and Dynamical Systems, vol.23, pp. 327-370, 2015.

[10] S. Biswas , M. Saifuddin, S.K. Sasmal, S. Samanta, N. Pal, F. Ababneh, J. Chattopadhyay, “A delayed prey-predator system with prey subject to the strong Allee effect and disease", Nonlinear Dyn., vol.84, no. 3, 1569-1594, 2016.

[11] S. Biswas, S.K. Sasmal, S. Samanta, M. Saifuddin, N. Pal, J. Chattopadhyay, "Optimal harvesting and complex dynamics in a delayed eco-epidemiological model with weak Allee effects", Nonlinear Dyn., vol. 87, no. 3, pp.1553-1573, 2017.

[12] K. Ghosh, S. Biswas, S. Samanta, P.K. Tiwari, A.S. Alshomrani, J. Chattopadhyay, "Effect of multiple delays in an eco-epidemiological model with strong Allee effect", Inter. J. Bifur. Chaos, vol. 27 , no. 11, 1750167, 2017.

[13] Hiba A. and Raid K.N., "A prey- predator model with Michael Mentence type of predator harvesting and infectious disease in prey", Iraqi Journal of Science, vol. 61, no. 5, pp. 1146$1163,2020$.

[14] P. Leslie and J. Gower. "The properties of a stochastic model for the predator-prey type of interaction between two species". Biometrika, vol. 47, pp. 219-234, 1960.

[15] R. May. Stability and complexity in model ecosystems, volume 6 of Monographs in population biology. Princeton University Press, Princeton, N.J., 1974.

[16] C. Arancibia-Ibarra and E. González-Olivares. "A modified Leslie-Gower predator-prey model with hyperbolic functional response and Allee effect on prey". BIOMAT 2010 International Symposium on Mathematical and Computational Biology, pages 146-162, 2011.

[17] M. Aziz-Alaoui and M. Daher. "Boundedness and global stability for a predator-prey model with modified Leslie-Gower and Holling-type II schemes". Applied Mathematics Letters, vol.16, pp.1069-1075, 2003.

[18] P. Feng and Y. Kang. "Dynamics of a modified Leslie-Gower model with double Allee effects". Nonlinear Dynamics, vol. 80, pp. 1051-1062, 2015.

[19] A. Singh and S. Gakkhar. "Stabilization of modified Leslie-Gower prey-predator model". Differential Equations and Dynamical Systems, vol. 22, pp. 239-249, 2014.

[20] Tchuenche, J.M., Dube, N., Bhunu, C.P. et al. "The impact of media coverage on the transmission dynamics of human influenza". BMC Public Health 11, S5 (2011). https://doi.org/10.1186/14712458-11-S1-S5.

[21] Liu Y, Cui J, "The impact of media coverage on the dynamics of infection disease". Int. J. Biomath., vol. 1, pp. 65-74, 2008.

[22] J. M. Tchuenche and C. T. Bauch. "Dynamics of an Infectious Disease Where Media Coverage Influences Transmission". International Scholarly Research Notices, vol. (2012) . |Article ID 581274 | https://doi.org/10.5402/2012/581274.

[23] Li Y, Cui J., " The effect of constant and pulse vaccination on SIS epidemic models incorporating media coverage" Commun. Nonlinear Sci. Numer. Simulat., vol. 14, pp. 2353-2365, 2009.

[24] W. Alwan and H. Abdul satar, "the Effect of Media Coverage on the Dynamics of Disease in Prey-Predator Model”, Iraqi Journal of Science, vol. 62, no. 3, pp. 981-996, 2021.

[25] Freedman H.I. and Waltman P., "Persistence in models of three interacting predator prey populations", Mathematical Bioscience, vol. 68, pp. 213-231, 1984.

[26] Perko L., Differential equations and Dynamical system, Third Edition, Springer-Verlag, New York. Inc., 2001. 